\title{
ENDPOINT MAPPING PROPERTIES OF SPHERICAL MAXIMAL OPERATORS
}

\author{
Andreas Seeger Terence Tao James Wright
}

\section{Introduction}

For a function $f \in L^{p}\left(\mathbb{R}^{d}\right), d \geq 2$, we consider the spherical means

$$
\mathcal{A}_{t} f(x)=\int_{S^{d-1}} f(x-t y) d \sigma(y)
$$

where $d \sigma$ is the rotationally invariant measure on $S^{d-1}$, normalized such that $\sigma\left(S^{d-1}\right)=1$. We wish to study the question of pointwise convergence as $t \rightarrow 0$ where the radii $t$ are restricted to a subset $E$ of $(0, \infty)$. Pointwise convergence is established from boundedness properties of the maximal function

$$
\mathcal{M}_{E} f(x)=\sup _{t \in E}\left|\mathcal{A}_{t} f(x)\right|
$$

for $f \in L^{p}\left(\mathbb{R}^{d}\right)$.

Stein [14] showed that for $E=\mathbb{R}_{+}$the maximal operator $\mathcal{M}_{E}$ is bounded on $L^{p}$ if and only if $p>$ $d /(d-1), d \geq 3$; the same result for the case $d=2$ was later proved by Bourgain [2]. The critical exponent $p(E)$ for $L^{p}$ boundedness of $\mathcal{M}_{E}$, for any set $E \in(0, \infty)$, was determined by Seeger, Wainger and Wright [12]. It is computed using a dilation invariant notion of Minkowski-dimension. In order to describe the result we let $N(E, \delta)$ be the $\delta$-entropy number of $E$, that is the minimal number of intervals of length $\delta$ needed to cover $E$ (we shall always redefine $N(\emptyset, \delta)=1$ ). Define

$$
E^{k}=\left[2^{k}, 2^{k+1}\right) \cap E
$$

and

$$
p(E)=1+\frac{1}{d-1}\left(\sup _{\delta>0} \sup _{k \in \mathbb{Z}} \frac{\log N\left(E^{k}, 2^{k} \delta\right)}{\log \delta^{-1}}\right) .
$$

Then $\mathcal{M}_{E}$ is bounded on $L^{p}$ for $p>p(E)$ and unbounded on $L^{p}$ if $p<p(E)$. Moreover various $L^{p}$ results were proven in [12] for the critical exponent $p=p(E)$; however these results fell short of being necessary and sufficient.

For the case that our maximal operator acts only on radial functions sharp endpoint estimates in almost all cases have been obtained in [13]. The relevant condition for $1<p<d /(d-1)$ turned out to be

Condition $\left(\mathcal{C}_{p, q}\right)$.

$$
\begin{array}{ll}
\sup _{j}\left(\sum_{n \geq 0}\left[N\left(E^{j+n}, 2^{j}\right)\right]^{q / p} 2^{-n(d-1) q / p^{\prime}}\right)^{1 / q}<\infty & \text { if } p \leq q<\infty, \\
\sup _{\substack{k \in \mathbb{Z} \\
\delta>0}} N\left(E^{k}, 2^{k} \delta\right)^{1 / p} \delta^{(d-1) / p^{\prime}}<\infty & \text { if } q=\infty .
\end{array}
$$

The first author is supported in part by a grant from the National Science Foundation. The second author is a Clay Prize fellow and is supported by the Sloan and Packard foundations.

Typeset by $\mathcal{A M S}_{\mathcal{S}}-\mathrm{T}_{\mathrm{EX}}$ 
It is shown in [13] that for $\mathcal{M}_{E}$ to map $L_{\text {rad }}^{p}$ to the Lorentz space $L^{p, q}, 1<p<d /(d-1), p \leq q \leq \infty$ it is necessary and sufficient that condition $\left(\mathcal{C}_{p, q}\right)$ holds. The necessity can be shown by testing $\mathcal{M}_{E}$ on characteristic functions of small balls. Observe that $\left(\mathcal{C}_{p, \infty}\right)$ is the limiting case of $\left(\mathcal{C}_{p, q}\right)$ as $q \rightarrow \infty$. For $p=d /(d-1)$ there are different characterizations for $L_{\text {rad }}^{p} \rightarrow L^{p, q}$ boundedness, at least when $d>2$.

The main purpose of this paper is to prove analogues of the $L_{\mathrm{rad}}^{p} \rightarrow L^{p}$ and $L_{\mathrm{rad}}^{p} \rightarrow L^{p, \infty}$ endpoint estimates for general functions in $L^{p}$, assuming however an additional regularity assumption (see hypothesis $\left(\mathcal{R}_{p}\right)$ below). The main general results for $1<p \leq d /(d-1)$ are stated in Theorem I, II, III and IV below. The case where each set $E^{k}=E \cap\left[2^{k}, 2^{k+1}\right]$ is a convex sequence serves as a model case (see $\S 8$ below). In particular we have

\section{Theorem 1.1.}

(i) Let $0<\alpha<\infty$ and let

$$
E(\alpha)=\left\{2^{k}\left(1+\nu^{-\alpha}\right): k \in \mathbb{Z}, \nu \in \mathbb{Z}^{+}\right\}
$$

Then $M_{E(\alpha)}$ is of weak type $(p, p)$ if and only if $p \geq 1+[(d-1)(\alpha+1)]^{-1}$.

(ii) Let $1 /(d-1)<\beta<\infty$ and let

$$
\widetilde{E}(\beta)=\left\{2^{k}\left(1+\log ^{-\beta}(2+\nu)\right): k \in \mathbb{Z}, \nu \in \mathbb{Z}^{+}\right\} .
$$

Then $M_{\widetilde{E}(\beta)}$ is of weak type $(p, p)$ if and only if $p \geq d /(d-1)$.

Remarks.

(a) Only the endpoint cases $p=1+[(d-1)(\alpha+1)]^{-1}$ and $p=d /(d-1)$ are new. When $\beta<1 /(d-1)$, $M_{\widetilde{E}(\beta)}$ fails to be weak type $(d /(d-1), d /(d-1))$. The case $\beta=1 /(d-1)$ remains open.

(b) For $p=1$ a slight variant was obtained by M. Christ who proved that the lacunary spherical maximal operator (with $E=\left\{2^{k}: k \in \mathbb{Z}\right\}$ ) maps the Hardy space $H^{1}$ to $L^{1, \infty}$. This can be deduced from a simple modification of the proof below, and in fact the weak type estimates in $\S 5$ are extensions of Christ's argument.

(c) It is not known whether the lacunary spherical maximal function maps $L^{1}$ to $L^{1, \infty}$. The closest known result is a weak type $L \log \log L$ inequality proved by the authors in [11].

We shall now formulate a technical result on $L^{p}$ boundedness for $\mathcal{M}_{E}$ which is only a minor improvement of the result in [12]. It gives a reasonably sharp but not yet definitive estimate for general sets $E$ of dilations. It will be applied however to sets which tend to be much thinner than the original sets.

Proposition 1.2. Suppose that $d \geq 2$ and $1<p \leq d /(d-1)$. Suppose that $\left\{\omega_{j}\right\}_{j=0}^{\infty}$ is a sequence of positive numbers satisfying

$$
\sum_{j \geq 0} \omega_{j}^{-p^{\prime}} \leq 1
$$

and suppose that

$$
\sup _{k \in \mathbb{Z}} \sum_{j \geq 0} \omega_{j}^{p} N\left(E^{k+j}, 2^{k}\right) 2^{-j(d-1) p / p^{\prime}} \leq A_{0}^{p}
$$

Then $\mathcal{M}_{E}$ is bounded on $L^{p}\left(\mathbb{R}^{d}\right)$, with operator norm dominated by $C A_{0}$.

We now describe our regularity assumption and begin with the following 
Definition. (i) A set $J \subset \mathbb{R}^{+}$is equally spaced with width $\delta$ and possible deviation $C>1$ if for all $t \in J$ the inequalities

$$
C^{-1} \delta \leq \operatorname{dist}(t, J \backslash\{t\}) \leq C \delta
$$

hold.

(ii) A family $\mathcal{J}=\{J\}$ of subsets of $\mathbb{R}^{+}$is uniformly equally spaced if for every $J \in \mathcal{J}$ there is a $\delta=\delta(J)>0$ so that $(1.8)$ holds with $\delta(J)$ and a constant $C$ independent of $J$. of $J$.

(iii) Let $J$ be an equally spaced subset of $\mathbb{R}^{+}$. Then we call $a_{J}=\inf J$ and $b_{J}=\sup J$ the endpoints

(iv) Let $\mathcal{J}$ be uniformly equally spaced family of subsets of $\mathbb{R}^{+}$. Then we denote by $\mathcal{D}(\mathcal{J})$ the set of endpoints $\mathcal{D}(\mathcal{J})=\cup_{J \in \mathcal{J}}\left\{a_{J}, b_{J}\right\}$.

Our regularity assumption will say that each $E^{k}$ can be split into "not too many" equally spaced sets. This gives a large class of examples, since in general the sets $\mathcal{D}^{k}$ of endpoints are often much thinner than the sets $E^{k}$.

\section{Regularity hypothesis $\left(\mathcal{R}_{p}\right)$.}

E satisfies hypothesis $\left(\mathcal{R}_{p}\right)$ if for each $k$ there is a collection $\mathcal{J}^{k}=\{J\}$ of subsets of $\left[2^{k}, 2^{k+1}\right]$ such that $E^{k} \subset \cup_{J \in \mathcal{J}^{k}} J$ and the following three conditions are satisfied.

(a) The family $\left\{J: J \in \cup_{k \in \mathbb{Z}} \mathcal{J}^{k}\right\}$ is uniformly equally spaced (with uniform possible deviation $C$ ).

(b) There is a positive sequence $\omega=\left\{\omega_{j}\right\}_{j=0}^{\infty}$ with $\sum_{j=0}^{\infty} \omega_{j}^{-p^{\prime}} \leq 1$ so that the sets of endpoints $\mathcal{D}^{k} \equiv$ $\mathcal{D}\left(\mathcal{J}^{k}\right)=\cup_{J \in \mathcal{J}^{k}}\left\{a_{J}, b_{J}\right\}$ satisfy

$$
\sup _{k \geq 0}\left(\sum_{j \geq 0}\left[N\left(\mathcal{D}^{k+j}, 2^{k}\right) 2^{-j(d-1) p / p^{\prime}} \omega_{j}^{p}\right)^{1 / p} \leq C_{0}<\infty .\right.
$$

(c) Let $\mathcal{J}_{\mu}^{k}$ denote the subfamily of all $J \in \mathcal{J}^{k}$ which are equally spaced with width $2^{k-\mu}$ and possible deviation $C$. Then we assume that there is $C_{1}>1$ such that

$$
\sum_{J \in \mathcal{J}_{\mu}^{k}} \operatorname{card}(J) \leq C_{1} N\left(E^{k}, 2^{k-\mu}\right)
$$

for every $k \in \mathbb{Z}, \mu \in \mathbb{N}$.

Note that by Proposition 1.2 the hypothesis $\left(\mathcal{R}_{p}\right)$ insures that the maximal operator associated to the set of endpoints, $\cup_{k>0} \mathcal{D}^{k}$, maps $L^{p}$ to $L^{p}$.

Our main results are

Theorem I. Suppose that $1<p<d /(d-1)$ and suppose that $E$ satisfies the regularity assumption $\left(\mathcal{R}_{p}\right)$. Then $\mathcal{M}_{E}$ is bounded on $L^{p}\left(\mathbb{R}^{d}\right)$ if and only if condition $\left(\mathcal{C}_{p, p}\right)$ holds.

Theorem II. Suppose that $1<p<d /(d-1)$, and suppose that $E$ satisfies the regularity assumption $\left(\mathcal{R}_{p}\right)$. Then $\mathcal{M}_{E}$ is of weak type $(p, p)$ if and only if condition $\left(\mathcal{C}_{p, \infty}\right)$ holds.

It is well known that the weak type $(p, p)$ bounds imply pointwise convergence theorems. By the Theorems of Calderón and Stein $[15$, ch.X, $\S 2]$ and the fact that $\left(\mathcal{C}_{p, \infty}\right)$ is necessary for the $L^{p} \rightarrow L^{p, \infty}$ inequality, these are sharp: 
Corollary 1.3. Let $\left\{t_{j}\right\}_{j=1}^{\infty}$ be a sequence with $\lim _{j \rightarrow \infty} t_{j}=0$ and assume that $E=\left\{t_{j}\right\}$ satisfies condition $\left(\mathcal{R}_{p}\right)$ for some $p \in(1, d /(d-1))$. Let $\mathcal{A}_{t_{j}} f(x)=\int_{S^{d-1}} f\left(x-t_{j} y^{\prime}\right) d \sigma\left(y^{\prime}\right)$.

(i) Suppose that $E$ satisfies condition $\left(\mathcal{C}_{p, \infty}\right)$. Then $\lim _{j \rightarrow \infty} \mathcal{A}_{t_{j}} f(x)=f(x)$ almost everywhere.

(ii) If condition $\left(\mathcal{C}_{p, \infty}\right)$ is not satisfied then there is a nonnegative function $f \in L^{p}\left(\mathbb{R}^{d}\right)$ such that $\lim \sup _{j \rightarrow \infty} \mathcal{A}_{t_{j}} f(x)=\infty$ almost everywhere.

Remark. It would of course be interesting to know whether some regularity assumption is needed. As a typical example where the regularity assumption fails consider the Cantor middle third set, translated by 1 , so that $E_{0}=\left\{1+\sum_{\nu=1}^{\infty} b_{\nu} 3^{-\nu}: b_{\nu} \in\{0,2\}\right\}$ and let $E=\cup_{k \in \mathbb{Z}} E_{0}$. Now the critical exponent is $p_{\text {cr }}=1+(d-1)^{-1} \log 2 / \log 3$. The set $E_{0}$ satisfies condition $\left(\mathcal{C}_{p_{\mathrm{cr}}, p_{\mathrm{cr}}}\right)$ and the set $E$ satifies condition $\left(\mathcal{C}_{p_{\text {cr }}, \infty}\right)$. However $\mathcal{R}_{p_{\text {cr }}}$ fails to hold and thus Theorems I and II above do not apply. It is not known whether $\mathcal{M}_{E_{0}}$ or $\mathcal{M}_{E}$ are of weak type $\left(p_{\mathrm{cr}}, p_{\mathrm{cr}}\right)$; see however a counterexample for a closely related maximal operator in $\S 8.2$ below. A much easier result is that $\mathcal{M}_{E}$ is of restricted weak type, see Proposition 1.4 below.

We now turn to the limiting case $p=p_{d}:=d /(d-1)$. There are sharp results, at least for $L^{p_{d}}$ boundedness, although conditions $\left(\mathcal{R}_{p}\right)$ and $\left(\mathcal{C}_{p, p}\right)$ are replaced by the following different conditions $\left(\widetilde{\mathcal{R}}_{p_{d}}\right)$ and $\left(\widetilde{\mathcal{C}}_{p_{d}}\right)$, respectively.

Regularity hypothesis $\left(\widetilde{\mathcal{R}}_{p_{d}}\right)$.

E satisfies hypothesis $\left(\widetilde{\mathcal{R}}_{p_{d}}\right)$ if for each $k$ there is a collection $\mathcal{J}^{k}=\{J\}$ of subsets of $\left[2^{k}, 2^{k+1}\right]$ so that assumptions (a) and (b) in $\left(\mathcal{R}_{p}\right)$ hold but (c) in $\left(\mathcal{R}_{p}\right)$ is replaced by

( $\widetilde{c})$ There is a $C_{1}>1$ such that

$$
\sum_{\mu \geq n} 2^{-\mu} \sum_{J \in \mathcal{J}_{\mu}^{k}} \operatorname{card}(J) \leq C_{1} 2^{-n} N\left(E^{k}, 2^{k-n}\right)
$$

holds uniformly in $n \in \mathbb{N}$.

The analogue of condition $\left(\mathcal{C}_{p, p}\right)$ is

\section{Condition $\left(\widetilde{\mathcal{C}}_{p_{d}}\right)$.}

The discrete measure $\sum_{k \in \mathbb{Z}} \sum_{n>0} N\left(E^{k}, 2^{k-n}\right) 2^{-n} n^{1 /(d-1)} \delta_{k, n}$ is a Carleson measure on the upper half plane; i.e.

$$
\sup _{|I| \geq 1} \frac{1}{|I|} \sum_{(k, n) \in T(I)} N\left(E^{k}, 2^{k-n}\right) 2^{-n} n^{1 /(d-1)}<\infty
$$

where the supremum is taken over all intervals of length $\geq 1$ and $T(I)$ is the tent of $I$, i.e. $T(I)=\{(x, t)$ : $x \in I, 0 \leq t \leq|I|\}$.

It was shown in [13] that for $d \geq 3$ condition $\left(\widetilde{\mathcal{C}}_{p_{d}}\right)$ is equivalent with the $L^{p_{d}}$ boundedness of $\mathcal{M}_{E}$ on radial functions. For general $L^{p}$ functions we have a similar result provided that hypothesis $\left(\widetilde{\mathcal{R}}_{p_{d}}\right)$ is satisfied:

Theorem III. Let $d \geq 2$ and $p_{d}=d /(d-1)$ and suppose that $E$ satisfies the regularity assumption $\left(\widetilde{\mathcal{R}}_{p_{d}}\right)$. Then $\mathcal{M}_{E}$ is bounded on $L^{p_{d}}\left(\mathbb{R}^{d}\right)$ if and only if condition $\left(\widetilde{\mathcal{C}}_{p_{d}}\right)$ holds.

Concerning a weak type $\left(p_{d}, p_{d}\right)$ inequality in dimensions $d \geq 3$ one may conjecture that the hypothesis

$$
N\left(E^{k}, 2^{k} \delta\right) \leq C \delta^{-1}[\log (1 / \delta)]^{-1 /(d-1)}
$$

is necessary and sufficient for $L^{p_{d}} \rightarrow L^{p_{d}, \infty}$ boundedness as this is shown to hold in [13] on $L_{\text {rad }}^{p_{d}}$. For general functions $f$ and under the regularity assumption $\left(\widetilde{\mathcal{R}}_{p_{d}}\right)$ we prove the following slightly weaker result. 
Theorem IV. Let $d \geq 3$ and $p_{d}=d /(d-1)$ and suppose that $E$ satisfies the regularity assumption $\left(\widetilde{\mathcal{R}}_{p_{d}}\right)$ and suppose that

$$
N\left(E^{k}, 2^{k} \delta\right) \leq C \delta^{-1}[\log (1 / \delta)]^{-1 /(d-1)}[\log \log (1 / \delta)]^{-1}
$$

uniformly in $k \in \mathbb{Z}$ and $\delta \leq e^{-2}$. Then $\mathcal{M}_{E}$ is of weak type $\left(p_{d}, p_{d}\right)$.

At present we do not know whether the same conclusion holds under the weaker condition (1.13). This accounts for the as yet undecided weak type $\left(p_{d}, p_{d}\right)$ estimate for $M_{\widetilde{E}(\beta)}$ in the remaining case $\beta=1 /(d-1)$ in Theorem 1.1.

We now briefly turn to the question of restricted weak type inequalities. Here no regularity assumption is needed.

Proposition 1.4. Let $1<p \leq d /(d-1), d \geq 3$ or $1<p<2, d=2$ and suppose that $E$ satisfies condition $\left(\mathcal{C}_{p, \infty}\right)$. Then $\mathcal{M}_{E}$ is of restricted weak type $(p, p)$, i.e. it maps $L^{p, 1}$ to $L^{p, \infty}$.

It remains open whether for the range $1<p<d /(d-1)$ the operator is of weak type $(p, p)$, under condition $\left(\mathcal{C}_{p, \infty}\right)$ alone, without the regularity assumption. Proposition 1.4 is much more straightforward than Theorem II above and we shall not give the details of the proof here. For $p_{d}=d /(d-1), d \geq 3$ the result had been already proved by Bourgain [1], and a variant of his argument applies for $1<p<d /(d-1)$ as well. Indeed let $\mathcal{A}_{t}^{j}$ be the frequency localized operator as in (2.1) below and define the maximal operator $M_{j}$ by $M_{j} f(x)=\sup _{t \in E}\left|\mathcal{A}_{t}^{j} f(x)\right|$. Then the estimates in [12] show that for $1<q \leq 2$ the operator $M_{j}$ is bounded on $L^{q}$ with norm $O\left(2^{j(d-1)\left(\frac{1}{q^{\prime}}-\frac{p-1}{q}\right)}\right)$ and the argument in [1] shows the restricted weak type estimate. The argument fails for $p=d=2$ and in fact the question whether the full circular maximal function is of restricted weak type $(2,2)$ (i.e. maps $L^{2,1}$ to $L^{2, \infty}$ ) had been posed in [16]. We note that Leckband [7] proved that for radial functions one has indeed $L_{\mathrm{rad}}^{2,1} \rightarrow L^{2, \infty}$ boundedness. However a Besicovitch set construction can be used to disprove the restricted weak type $(2,2)$ inequality for general functions. The argument (see $\S 8$ below) shows

Proposition 1.5. Suppose $d=2$ and

$$
\sup _{k>0} \sup _{\delta<1 / 10} N\left(E^{k}, 2^{k} \delta\right) \delta \log \delta^{-1}=\infty .
$$

Then $\mathcal{M}_{E}$ is not of restricted weak type $(2,2)$.

Structure of the paper: In $\S 2$ we shall review some essentially known estimates for spherical means which are needed later. In $\S 3$ we shall review atomic decompositions in $L^{p}$. $\S 4$ contains a proof of the $L^{p}$ estimates as stated in Proposition 1.2 and Theorem I. The weak type $(p, p)$ inequalities (Theorem II) are proved in $\S 5$. The necessary modifications for the proofs of Theorem III and IV are discussed in $\S 6$ and $\S 7$, respectively. In $\S 8$ we discuss some examples and include the proof of Proposition 1.5.

\section{Estimates on spherical means}

We shall need to introduce regularizations of $\mathcal{A}_{t}$ in (1.1) via dyadic frequency cutoffs. Let $\beta_{0}$ be a radial $C_{0}^{\infty}$ function so that $\beta_{0}(\xi)=1$ if $|\xi| \leq 1$ and $\beta_{0}(\xi)=0$ if $|\xi| \geq 2$. For $j=1,2, \ldots$ let $\beta_{j}(\xi)=\beta_{0}\left(2^{-j} \xi\right)-\beta_{0}\left(2^{1-j} \xi\right)$ and define $\mathcal{A}_{t}^{j}$ by

$$
\widehat{\mathcal{A}_{t}^{j}} f(\xi)=\widehat{d \sigma}(t \xi) \beta_{j}(t \xi) \widehat{f}(\xi)
$$

so that

$$
\mathcal{A}_{t}=\sum_{j=0}^{\infty} \mathcal{A}_{t}^{j} .
$$


Let $\tilde{\beta}$ be a radial $C_{0}^{\infty}$ function which is supported where $2^{-6} \leq|\xi| \leq 2^{6}$ and equal to 1 when $2^{-5} \leq$ $|\xi| \leq 2^{5}$. Let $P^{l} f$ be defined by $\widehat{P^{l} f}(\xi)=\tilde{\beta}\left(2^{-l} \xi\right) \widehat{f}(\xi)$ and observe that

$$
\mathcal{A}_{t}^{j} f=\mathcal{A}_{t}^{j} P^{j-k} f \quad \text { if } t \in E^{k} .
$$

Clearly the maximal function $\sup _{t>0}\left|\mathcal{A}_{t}^{j} f(x)\right|$ is dominated by $C_{j} M_{H L} f(x)$ where $M_{H L}$ is the HardyLittlewood maximal function of $f$; in fact $C_{j}=O\left(2^{j}\right)$ (cf. Lemma 2.1 below). Therefore

$$
\mathcal{M}_{E} f(x) \leq M_{H L} f(x)+\sup _{k \in \mathbb{Z}} \sup _{t \in E^{k}}\left|\sum_{j \geq 10} \mathcal{A}_{t}^{j} P_{j-k} f(x)\right|
$$

and throughout this paper we shall assume that summations in $j$ are extended over $j \geq 10$.

Here we collect well known estimates on spherical means and its regularization $\mathcal{A}_{t}^{j}$ which were used in this or a related form in previous papers (in particular see [12] for some of the more technical statements).

Lemma 2.1. Let $\mathcal{A}_{t}^{j}$ be as above and let $B_{t}^{j}=\frac{d}{d t} \mathcal{A}_{t}^{j}$. Suppose that $2^{k} \leq t \leq 2^{k+1}, j \geq 10$ and that $1 \leq p \leq 2$.

(i)

$$
\left|\mathcal{A}_{t}^{j} f(x)\right|+2^{-j} t\left|B_{t}^{j} f(x)\right| \leq C_{M} 2^{j} \int \frac{t^{-d}}{\left(1+2^{j}\left|\frac{|x-y|}{t}-1\right|\right)^{M}}|f(y)| d y
$$

$$
\left\|\mathcal{A}_{t}^{j}\right\|_{L^{p} \rightarrow L^{p}}+2^{k-j}\left\|B_{t}^{j}\right\|_{L^{p} \rightarrow L^{p}} \lesssim 2^{-j(d-1) / p^{\prime}} .
$$

(iii) Let $I \subset\left[2^{k-1}, 2^{k+2}\right]$ be an interval of length $2^{k-j}$. Then

$$
\left\|\sup _{t \in I}\left|\mathcal{A}_{t}^{j} f\right|\right\|_{L^{p}} \lesssim 2^{-j(d-1) / p^{\prime}}\|f\|_{L^{p}}
$$

Sketch of Proof. (i) is a straightforward calculation, which also implies (ii) for $p=1$. It is well known that $|\widehat{d \sigma}(\xi)| \lesssim(1+|\xi|)^{-(d-1) / 2}$ and thus (ii) for $p=2$ follows, and interpolation settles the case $1<p<2$. (iii) follows by writing $\mathcal{A}_{t}^{j}=\mathcal{A}_{t_{0}}^{j}+\int_{t_{0}}^{t} B_{s}^{j} d s$ for $t_{0} \in I$.

Definition. For a set $\mathcal{E}$ of dilations and $L \in \mathbb{Z}$, let $\mathfrak{I}_{L}(\mathcal{E})$ be a minimal collection of dyadic intervals of length $2^{L}$ covering $\mathcal{E}$. For $I \in \mathfrak{I}_{L}(\mathcal{E})$ let $r_{I}$ denote the midpoint of the interval $I$, and for a dyadic cube $Q$, let $2^{L(Q)}$ denote its sidelength. Then for $\eta \geq 1$, we define

$$
V_{Q, \eta}(\mathcal{E})=\bigcup_{I \in \mathfrak{I}_{L(Q)}(\mathcal{E})}\left\{x \in \mathbb{R}^{d}:|| x-x_{Q}\left|-r_{I}\right| \leq 2^{L(Q)+4} \eta\right\}
$$

for $\eta=1$ we also write $V_{Q}(\mathcal{E})=V_{Q, 1}(\mathcal{E})$.

Lemma 2.2. Let $\mathcal{E} \subset\left[2^{k}, 2^{k+1}\right]$.

(i) For $1 \leq p \leq 2$,

$$
\left\|\sup _{t \in \mathcal{E}}\left|\mathcal{A}_{t}^{j} f\right|\right\|_{L^{p}} \lesssim\left[N\left(\mathcal{E}, 2^{k-j}\right)\right]^{1 / p} 2^{-j(d-1) / p^{\prime}}\|f\|_{L^{p}}
$$

(ii) Let $Q$ be a dyadic cube, let $f_{Q}$ be an $L^{2}$ function supported on $Q$ and suppose $k-j \leq L(Q) \leq k-10$. Then

$$
\left\|\sup _{t \in \mathcal{E}}\left|\mathcal{A}_{t}^{j} f_{Q}\right|\right\|_{L^{1}\left(V_{Q}(\mathcal{E})\right)} \lesssim 2^{(-L(Q)+k-j)(d-1) / 2} N\left(\mathcal{E}, 2^{k-j}\right) 2^{L(Q) d / 2}\left\|f_{Q}\right\|_{L^{2}}
$$


(iii) Let $\mathcal{Q}$ be a collection of pairwise disjoint cubes of sidelength $2^{k-j+\sigma}$ where $\sigma \geq 0$. Then for $\sigma \leq j$,

$$
\left\|\sup _{t \in \mathcal{E}}\left|\mathcal{A}_{t}^{j}\left[\sum_{Q \in \mathcal{Q}} f_{Q}\right]\right|\right\|_{L^{p}} \lesssim 2^{-\sigma(d-1)(1 / p-1 / 2)}\left[N\left(\mathcal{E}, 2^{k-j}\right)\right]^{1 / p} 2^{-j(d-1) / p^{\prime}}\left(\sum_{Q \in \mathcal{Q}}|Q|^{1-p / 2}\left\|f_{Q}\right\|_{L^{2}}^{p}\right)^{1 / p}
$$

(iv) Let $\mathcal{Q}$ be as in (iii) and let $\mathcal{V}$ be an open set containing $\bigcup_{Q \in \mathcal{Q}} V_{Q, \eta}(\mathcal{E})$. Then, for $\eta \geq 1$,

$$
\left\|\sup _{t \in \mathcal{E}}\left|\mathcal{A}_{t}^{j}\left[\sum_{Q \in \mathcal{Q}} f_{Q}\right]\right|\right\|_{L^{p}\left(\mathbb{R}^{d} \backslash \mathcal{V}\right)} \leq C_{M}\left(2^{\sigma} \eta\right)^{-M\left(\frac{2}{p}-1\right)}\left[N\left(\mathcal{E}, 2^{k-j}\right)\right]^{1 / p} 2^{-j(d-1) / p^{\prime}}\left(\sum_{Q \in \mathcal{Q}}\left\|f_{Q}\right\|_{L^{p}}^{p}\right)^{1 / p} .
$$

(v) The estimates in (i), (ii), (iii) and (iv) remain valid if for $t \in \mathcal{E}$ the operator $\mathcal{A}_{t}^{j}$ is replaced by $2^{k-j} B_{t}^{j}=2^{k-j} \frac{d}{d t} \mathcal{A}_{t}^{j}$.

Sketch of Proof. (i) is a rather straightforward consequence of Lemma 2.1, (iii). To prove (ii) we use Cauchy-Schwarz to pass from an $L^{1}$ estimate on the exceptional set $V_{Q}(\mathcal{E})$ to an $L^{2}$ estimate (namely Lemma 2.1 (ii) with $p=2$ ), and for the estimate off the exceptional set we use the explicit form (2.4). (iv) for $p=2$ is a consequence of (i), and (iv) for $p=1$ follows from the explicit form of the kernel in Lemma 2.1 (i). The general case is obtained by interpolation. (iii) for $p=2$ is a consequence of (i), and (iii) for $p=1$ follows from (ii) and (iv). The general case is obtained by interpolation.

A small variant is

Lemma 2.3. Let $J \subset\left[2^{k}, 2^{k+1}\right]$ be an equally spaced set with width $2^{k-\mu}$ (here $\mu \geq 0$ ) and possible deviation $B$, and let $a_{J}<b_{J}$ be the endpoints of $J$. Suppose that $b_{J}-a_{J} \geq 2^{k-j}$ and $\mu \geq j$. Then the following statements hold.

(i)

$$
\left\|\sup _{t \in J}\left|\mathcal{A}_{t}^{j} f\right|\right\|_{L^{p}} \leq C_{B} N\left(J, 2^{k-\mu}\right)^{1 / p} 2^{-j(d-1) / p^{\prime}} 2^{(j-\mu) / p}\|f\|_{L^{p}}
$$

(ii) Let $\mathcal{Q}$ be a collection of pairwise disjoint cubes of sidelength $2^{k-j+\sigma}$ where $\sigma \geq 0$. Then for $\sigma \leq j$,

$$
\left\|\sup _{t \in J}\left|\mathcal{A}_{t}^{j}\left[\sum_{Q \in \mathcal{Q}} f_{Q}\right]\right|\right\|_{L^{p}} \leq C_{B} 2^{-\sigma(d-1)(1 / p-1 / 2)} \operatorname{card}(J)^{1 / p} 2^{-j(d-1) / p^{\prime}} 2^{(j-\mu) / p}\left(\sum_{Q \in \mathcal{Q}}|Q|^{1-p / 2}\left\|f_{Q}\right\|_{L^{2}}^{p}\right)^{1 / p} .
$$

(iii) Let $\mathcal{Q}$ be as in (ii) and let $\mathcal{V}$ be an open set containing $\bigcup_{Q \in \mathcal{Q}} V_{Q, \eta}(\mathcal{E})$. Then, for $\eta \geq 1$,

$$
\left\|\sup _{t \in J}\left|\mathcal{A}_{t}^{j}\left[\sum_{Q \in \mathcal{Q}} f_{Q}\right]\right|\right\|_{L^{p}\left(\mathbb{R}^{d} \backslash \mathcal{V}\right)} \leq C_{B, M}\left(2^{\sigma} \eta\right)^{-M\left(\frac{2}{p}-1\right)} \operatorname{card}(J)^{1 / p} 2^{(j-\mu) / p} 2^{-j(d-1) / p^{\prime}}\left(\sum_{Q \in \mathcal{Q}}\left\|f_{Q}\right\|_{L^{p}}^{p}\right)^{1 / p}
$$

Proof. We simply observe that if $b_{J}-a_{J} \geq 2^{k-j}$ then

$$
N\left(J, 2^{k-j}\right) \approx 2^{j-\mu} N\left(J, 2^{k-\mu}\right) \approx 2^{j-\mu} \operatorname{card}(J)
$$

and the conclusions (i)-(iii) follow from Lemma 2.2 . 


\section{Atomic decompositions}

We give a decomposition of the maximal operator and also the function it acts on; this is motivated by one of the proofs of the standard atomic decomposition (following [3], [9]) based on square functions; used for example in the theory of Hardy spaces on product domains.

For $c_{0}=10 \sqrt{d}$ let

$$
\mathcal{N}^{k} f(x)=\sup _{|y| \leq c_{0} 2^{-k}}\left|P^{k} f(x+y)\right|
$$

and define the maximal square function

$$
\mathcal{N} f(x)=\left(\sum_{k=-\infty}^{\infty}\left|\mathcal{N}^{k} f(x)\right|^{2}\right)^{1 / 2}
$$

then

$$
\|\mathcal{N} f\|_{L^{p}} \approx_{p}\|f\|_{L^{p}}, \quad 1<p<\infty
$$

and $\|\mathcal{N} f\|_{L^{1}} \approx\|f\|_{H^{1}}$.

Consider the level sets $\Omega_{n}=\left\{x: \mathcal{N} f(x)>2^{n}\right\}$ and the expanded sets $\widetilde{\Omega}_{n}=\left\{x: M_{H L} \chi_{\Omega}(x)>1 / 2\right\}$; here $M_{H L}$ is the Hardy-Littlewood maximal function. Then $\left|\widetilde{\Omega}_{n}\right| \leq C\left|\Omega_{n}\right|$. Let $\mathfrak{R}$ denote the family of all dyadic cubes and let $\mathfrak{R}_{n}$, for $n \in \mathbb{Z}$, denote the collection of all dyadic cubes $R$ with the property that $\left|R \cap \Omega_{n}\right|>|R| / 2$ but $\left|R \cap \Omega_{n+1}\right| \leq|R| / 2$. Then from these definitions one easily deduces

$$
\sum_{k=-\infty}^{\infty} \sum_{\substack{R \in \Re_{n} \\ L(R)=-k}}\left\|\left(P^{k} f\right) \chi_{R}\right\|_{L^{2}}^{2} \lesssim 2^{2 n}\left|\Omega_{n}\right|
$$

(see for example Lemma 3.1 in [9]).

Let $e_{R}=\left(P^{l} f\right) \chi_{R}$ if $L(R)=-l$. Then from (2.2) we have

$$
\mathcal{A}_{t}^{j} f=\mathcal{A}_{t}^{j} P^{j-k} f=\mathcal{A}_{t}^{j}\left[\sum_{L(R)=k-j} e_{R}\right]
$$

if $t \in E^{k}$.

Now $\widetilde{\Omega}_{n}$ is an open set with finite measure and we can form the Whitney-decomposition into dyadic cubes. Let $\mathfrak{W}_{n}$ be the set of Whitney cubes and observe that every $R \in \mathfrak{R}_{n}$ is contained in a unique Whitney-cube $Q(R)$. This defines a function $R \mapsto Q(R)$ for all dyadic cubes.

For a dyadic cube $Q$ we define now

$$
F_{Q}^{l}(f)=\sum_{\substack{Q(R)=Q \\ L(R)=-l}} e_{R}
$$

Notice that $F_{Q}^{l}=0$ if $-l>L(Q)$.

¿From (2.3) and (3.3), we have the pointwise estimate

$$
\mathcal{M}_{E} f(x) \lesssim M_{H L} f(x)+\sup _{k \in \mathbb{Z}} \sup _{t \in E^{k}} \sum_{j \geq 10}\left|\mathcal{A}_{t}^{j}\left[\sum_{L(Q) \geq k-j} F_{Q}^{j-k}(f)\right](x)\right| .
$$

It is useful to introduce a space $X^{p}$ of vector-valued functions as follows. 
Definition. Let $X^{p}$ be the space of vector-valued functions $F=\left(F_{Q}^{l}\right)$ where the dyadic cubes $Q$ satisfy $L(Q)+l \geq 0, F_{Q}^{l}$ is supported on $Q$, and

$$
\|F\|_{X^{p}}=\left(\sum_{Q}|Q|^{1-p / 2}\left(\sum_{l: L(Q)+l \geq 0}\left\|F_{Q}^{l}\right\|_{L^{2}}^{2}\right)^{p / 2}\right)^{1 / p}
$$

is finite.

We first observe

Lemma 3.1. For $1 \leq p \leq 2$,

$$
\|F(f)\|_{X^{p}} \lesssim\|\mathcal{N} f\|_{L^{p}}
$$

Proof. We write

$$
\begin{aligned}
\|F(f)\|_{X^{p}} & =\left(\sum_{Q}|Q|^{1-p / 2}\left(\sum_{\ell: L(Q)+\ell \geq 0}\left\|\sum_{\substack{Q(R)=Q \\
L(R)=-\ell}} e_{R}\right\|_{L^{2}}^{2}\right)^{p / 2}\right)^{1 / p} \\
& \leq\left(\sum_{Q}|Q|^{1-p / 2}\left(\sum_{\ell: L(Q)+\ell \geq 0} \sum_{\substack{R \in \Re_{n} \\
Q(R)=Q \\
L(R)=-\ell}}\left\|e_{R}\right\|_{L^{2}}^{2}\right)^{p / 2}\right)^{1 / p} .
\end{aligned}
$$

Now we use the imbedding $\ell^{p} \subset \ell^{2}$ for $p \leq 2$ to estimate the last expression by

$$
\begin{aligned}
& \left(\sum_{Q}|Q|^{1-p / 2} \sum_{n}\left(\sum_{\ell: L(Q)+\ell \geq 0} \sum_{\substack{R \in \Re_{n} \\
Q(R)=Q \\
L(R)=-\ell}}\left\|e_{R}\right\|_{L^{2}}^{2}\right)^{p / 2}\right)^{1 / p} \\
& \leq\left(\sum_{n} \sum_{Q \in \mathfrak{W}_{n}}|Q|^{1-p / 2}\left(\sum_{\substack{R \in \Re_{n} \\
Q(R)=Q}}\left\|e_{R}\right\|_{L^{2}}^{2}\right)^{p / 2}\right)^{1 / p}
\end{aligned}
$$

and by (3.2) and several applications of Hölder's inequality this in turn is estimated by

$$
\begin{aligned}
& \left(\sum_{n}\left(\sum_{Q \in \mathfrak{W}_{n}}|Q|\right)^{1-p / 2}\left(\sum_{Q} \sum_{\substack{R \in \mathfrak{R}_{n} \\
Q(R)=Q}}\left\|e_{R}\right\|_{L^{2}}^{2}\right)^{p / 2}\right)^{1 / p} \\
& \leq\left(\sum_{n}\left|\widetilde{\Omega}_{n}\right|^{1-p / 2}\left(\sum_{R \in \mathfrak{R}_{n}}\left\|e_{R}\right\|_{L^{2}}^{2}\right)^{p / 2}\right)^{1 / p} \\
& \lesssim\left(\sum_{n}\left|\widetilde{\Omega}_{n}\right|^{1-p / 2}\left(2^{2 n}\left|\Omega_{n}\right|\right)^{p / 2}\right)^{1 / p} \\
& \lesssim\left(\sum_{n}\left|\Omega_{n}\right| 2^{n p}\right)^{1 / p} \lesssim\|\mathcal{N} f\|_{L^{p}} .
\end{aligned}
$$

This proves the Lemma.

We now return to estimate the second term on the right of (3.5). The part where the sum extends over cubes $Q$ with $L(Q) \leq k$ is the most difficult to handle. In the following lemma we shall first dispose of the remaining part which is dealt with by straightforward $L^{2}$ estimates. 
Lemma 3.2. Let $1<p \leq 2, \varepsilon>0$ and suppose that

$$
\sup _{k} N\left(E^{k}, 2^{k} \delta\right)^{1 / 2} \delta^{(d-1-\varepsilon) / 2} \leq A .
$$

Let $\left\{\chi_{Q, l}\right\}$ be a family of measurable functions so that

$$
\sup _{l}\left\|\sum_{Q} \mid \chi_{Q, l}\right\|_{L^{2}} \leq 1
$$

and define

$$
\mathfrak{N}_{j} F(x)=\sup _{k \in \mathbb{Z}} \sup _{t \in E^{k}}\left|\mathcal{A}_{t}^{j}\left[\sum_{L(Q) \geq k} \chi_{Q, k} F_{Q}^{j-k}\right](x)\right|
$$

Then

$$
\left\|\mathfrak{N}_{j} F\right\|_{L^{p}} \leq C 2^{-\varepsilon j} A\|F\|_{X^{p}}
$$

where $C$ is independent of the choice of the particular family $\left\{\chi_{Q, k}\right\}$.

Proof. We shall verify (3.9) for $p=1$ and for $p=2$; the general case follows by interpolation.

For $p=2$ we replace the sup in $k$ by a square function and use Lemma 2.2 (i) to obtain

$$
\begin{aligned}
\left\|\mathfrak{N}_{j} F\right\|_{L^{2}} & \leq\left(\sum_{k}\left\|\sup _{t \in E^{k}} \mid \mathcal{A}_{t}^{j}\left[\sum_{\substack{Q: \\
L(Q) \geq k}} \chi_{Q, k} F_{Q}^{j-k}\right]\right\|_{L^{2}}^{2}\right)^{1 / 2} \\
& \leq C A 2^{-\varepsilon j}\left(\sum_{k}\left\|\sum_{\substack{Q: \\
L(Q) \geq k}} \chi_{Q, k} F_{Q}^{j-k}\right\|_{L^{2}}^{2}\right)^{1 / 2} \\
& \leq C A 2^{-\varepsilon j}\left(\sum_{k} \sum_{\substack{Q: \\
L(Q) \geq k}}\left\|F_{Q}^{j-k}\right\|_{L^{2}}^{2}\right)^{1 / 2}
\end{aligned}
$$

where for the last inequality we have used the assumption on the family $\left\{\chi_{Q, l}\right\}$. This proves (3.9) for $p=2$.

Now consider the case $p=1$. Given a cube $Q$ we let $Q^{*}$ denote the cube with same center but tenfold sidelength. We then estimate (following standard procedure in estimations of singular integrals acting on atoms)

$$
\left\|\mathfrak{N}_{j} F\right\|_{L^{1}} \leq \sum_{Q} I_{Q}+I I_{Q}
$$

where

$$
\begin{aligned}
I_{Q} & =\left\|\sup _{k} \sup _{t \in E^{k}} \mid \mathcal{A}_{t}^{j}\left[\chi_{Q, k} F_{Q}^{j-k}\right]\right\|_{L^{1}\left(Q^{*}\right)} \\
I I_{Q} & =\left\|\sup _{k} \sup _{t \in E^{k}} \mid \mathcal{A}_{t}^{j}\left[\chi_{Q, k} F_{Q}^{j-k}\right]\right\|_{L^{1}\left(\mathbb{R}^{d} \backslash Q^{*}\right)} .
\end{aligned}
$$

Now for $I_{Q}$ we use the Cauchy-Schwarz inequality and the $L^{2}$ estimate above to deduce that

$$
\begin{aligned}
I_{Q} & \lesssim|Q|^{1 / 2}\left\|\sup _{k} \sup _{t \in E^{k}} \mid \mathcal{A}_{t}^{j}\left[\chi_{Q, k} F_{Q}^{j-k}\right]\right\|_{L^{2}} \\
& \leq C A 2^{-\varepsilon j}|Q|^{1 / 2}\left(\sum_{k}\left\|F_{Q}^{j-k}\right\|_{L^{2}}^{2}\right)^{1 / 2}
\end{aligned}
$$


For $I I_{Q}$ we use Lemma 2.1 (i). In that formula we use that if $y \in Q, x \in Q^{*}, L(Q) \geq k, t \leq 2^{k+1}$ then $\left|t^{-1}\right| x-y|-1| \approx t^{-1}|x-y|$ and thus for $M>d \geq 2$

$$
\begin{aligned}
I I_{Q} & \lesssim 2^{j} \sum_{k \leq L(Q)} \int_{\left|x-y_{Q}\right| \geq 2^{L(Q)+2}} 2^{k(M-d)} 2^{-j M} \int_{Q}|x-y|^{-M}\left|F_{Q}^{j-k}(y)\right| d y d x \\
& \lesssim 2^{j(1-M)} \sum_{k \leq L(Q)} 2^{(k-L(Q))(M-d)}\left\|F_{Q}^{j-k}\right\|_{L^{1}(Q)} \\
& \lesssim 2^{j(1-M)}|Q|^{1 / 2}\left(\sum_{k}\left\|F_{Q}^{j-k}\right\|_{L^{2}}^{2}\right)^{1 / 2} .
\end{aligned}
$$

Now $M$ can be chosen to be $\geq 1+\varepsilon$ and we obtain that $\sum_{Q}\left(I_{Q}+I I_{Q}\right)$ is bounded by $C A 2^{-\varepsilon j}\|F\|_{X^{1}}$, thus proving (3.9) for $p=1$.

For the remainder of the paper we will only have to deal with the part in (3.5) dealing with the contribution $k>L(Q)$. Define for a positive integer $\sigma$

$$
\mathfrak{M}_{\sigma} F(x)=\sup _{k} \sup _{t \in E^{k}}\left|\sum_{j \geq \sigma} \mathcal{A}_{t}^{j}\left[\sum_{\substack{Q: \\ L(Q)=k-j+\sigma}} F_{Q}^{j-k}\right](x)\right| .
$$

Our main reduction in this section is

Proposition 3.3. Let $1<p<2$, suppose that hypothesis $\left(\mathcal{C}_{p, \infty}\right)$ is satisfied and suppose that for some $\varepsilon_{0}>0$ the inequality

$$
\left\|\mathfrak{M}_{\sigma} F\right\|_{L^{p, q}} \leq C_{0} 2^{-\varepsilon_{0} \sigma}\|F\|_{X^{p}}
$$

holds for all compactly supported $F$ (meaning that $F_{Q}^{l}$ vanishes for all but finitely many $l$ and $Q$ ). Then there is $c\left(p, \varepsilon_{0}\right)>0$ so that

$$
\left\|\mathcal{M}_{E} f\right\|_{L^{p, q}} \leq c\left(p, \varepsilon_{0}\right) C_{0}\|f\|_{L^{p}}
$$

for all $f \in L^{p}\left(\mathbb{R}^{d}\right)$.

Proof. Let $F_{Q}^{l}(f)$ be as in (3.4). For $\sigma=1,2, \ldots$ define $F_{\sigma}^{(1)}(f)$ by $\left[F_{\sigma}^{(1)}\right]_{Q}^{l}(f)=F_{Q}^{l}(f)$ if $L(Q)=\sigma-l$ and $\left[F_{\sigma}^{(1)}\right]_{Q}^{l}(f)=0$ if $L(Q) \neq \sigma-l$. For $j \geq 10$ define $\left[F_{j}^{(2)}\right]_{Q}^{l}(f)=F_{Q}^{j+l}(f)$ if $L(Q) \geq-l$ and zero otherwise and let $\chi_{Q, l}^{j}$ be the characteristic function of $\cup_{n \in \mathbb{Z}} \cup\left\{R: R \in \mathfrak{R}_{n}, L(R)=-l-j, Q(R)=Q\right\}$. Then for every fixed $j$ condition (3.7) is satisfied for the family $\left\{\chi_{Q, l}^{j}\right\}$.

¿From (3.5) we get

$$
\mathcal{M}_{E} f(x) \lesssim M_{H L} f(x)+\sum_{\sigma>0} \mathfrak{M}_{\sigma}\left[F_{\sigma}^{(1)}(f)\right](x)+\sum_{j \geq 10} \mathfrak{N}_{j}\left[F_{j}^{(2)}(f)\right](x)
$$

Note that it follows from Lemma 3.1 and (3.1) that $\left\|F_{\sigma}^{(1)}(f)\right\|_{X^{p}} \leq C_{p}\|f\|_{p}$ and $\left\|F_{j}^{(2)}(f)\right\|_{X^{p}} \leq C_{p}\|f\|_{p}$ for $1<p \leq 2$, uniformly in $\sigma$ and $j$. ¿From hypothesis $\left(\mathcal{C}_{p, \infty}\right)$ it follows that the assumption of Lemma 3.2 holds with $\varepsilon=(d-1)(2-p)$ which is positive since we are assuming $p<2$. Thus

$$
\left\|\sum_{j \geq 10} \mathfrak{N}_{j}\left[F_{j}^{(2)}(f)\right]\right\|_{L^{p, q}} \lesssim \sum_{j \geq 10} 2^{-\varepsilon j}\left\|F_{j}^{(2)}(f)\right\|_{X^{p}} \lesssim\|f\|_{p} .
$$

By our assumption we also have

$$
\left\|\sum_{\sigma>0} \mathfrak{M}_{\sigma}\left[F_{\sigma}^{(1)}(f)\right]\right\|_{L^{p, q}} \lesssim \sum_{\sigma>0} 2^{-\varepsilon_{0} \sigma}\left\|F_{\sigma}^{(1)}(f)\right\|_{X^{p}} \lesssim\|f\|_{p}
$$

and the proposition is proved. 


\section{4. $L^{p}$ estimates}

We shall use Proposition 3.3 and in order to prove $L^{p}$ estimates we have to verify the $X^{p} \rightarrow L^{p}$ estimate for $\mathfrak{M}_{\sigma}$ in (3.10). We shall first prove Proposition 1.2 where no regularity assumption is needed.

We shall also use the following definitions.

$$
G_{\sigma}^{l}(F)=\sum_{Q: L(Q)=-l+\sigma} F_{Q}^{l}
$$

and let $G_{\sigma}(F)=\left\{G_{\sigma}^{l}(F)\right\}_{l \in \mathbb{Z}}$ be the corresponding vector valued analogue.

Proposition 4.1. Suppose that $1<p<2$ and suppose that $\sum_{n} \omega_{n}^{-p^{\prime}} \leq 1$. Let

$$
\lambda_{j, k}=N\left(E^{k}, 2^{k-j}\right)^{1 / p} 2^{-j(d-1) / p^{\prime}} .
$$

Then

$$
\left\|\mathfrak{M}_{\sigma} F\right\|_{L^{p}} \lesssim 2^{-\sigma(d-1)(1 / p-1 / 2)} \sup _{l}\left(\sum_{n=0}^{\infty}\left|\omega_{n}\right|^{p} \lambda_{n, l+n}^{p}\right)^{1 / p}\|F\|_{X^{p}}
$$

Proof. We estimate using Hölder's inequality

$$
\left|\mathfrak{M}_{\sigma} F(x)\right| \lesssim\left(\sum_{k}\left\|\left\{\omega^{-1}\right\}\right\|_{\ell^{p^{\prime}}}^{p} \sum_{j=10}^{\infty} \omega_{j}^{p} \sup _{t \in E^{k}}\left|\mathcal{A}_{t}^{j} G_{\sigma}^{j-k}(F)\right|^{p}\right)^{1 / p}
$$

By Lemma 2.2 (iii) the $L^{p}$ norm of this expression is estimated as

$$
\begin{aligned}
\left\|\mathfrak{M}_{\sigma} F\right\|_{L^{p}} & \lesssim 2^{-\sigma(d-1)(1 / p-1 / 2)}\left(\sum_{k} \sum_{j}\left[\omega_{j} \lambda_{j, k}\right]^{p} \sum_{L(Q)=k-j+\sigma}|Q|^{1-p / 2}\left\|F_{Q}^{j-k}\right\|_{L^{2}}^{p}\right)^{1 / p} \\
& \lesssim 2^{-\sigma(d-1)(1 / p-1 / 2)}\left(\sum_{Q} \sum_{j}\left[\omega_{j} \lambda_{j, L(Q)+j-\sigma}\right]^{p}|Q|^{1-p / 2}\left\|F_{Q}^{\sigma-L(Q)}\right\|_{L^{2}}^{p}\right)^{1 / p} \\
& \lesssim 2^{-\sigma(d-1)(1 / p-1 / 2)}\left(\sum_{l} \sum_{Q: L(Q)=\sigma-l} \sum_{j}\left[\omega_{j} \lambda_{j, l+j}\right]^{p}|Q|^{1-p / 2}\left\|F_{Q}^{l}\right\|_{L^{2}}^{p}\right)^{1 / p} \\
& \lesssim 2^{-\sigma(d-1)(1 / p-1 / 2)}\|F\|_{X^{p}} .
\end{aligned}
$$

Proof of Proposition 1.2. Immediate from Propositions 3.3 and 4.1 when $1<p<2$. The case $p=2$ (and hence $d=2$ ) follows as in the proof of Proposition 4.1 where now we treat the whole operator $\mathcal{M}_{E}$.

We now turn to the proof of the

$L^{p}$ estimates under the regularity hypothesis. For the remainder of this section we shall fix a choice of $\mathcal{J}^{k}, \mathcal{J}_{\mu}^{k}$ as in the definition of regularity assumption $\left(\mathcal{R}_{p}\right)$.

Let $\sigma$ be a positive integer. Let

$$
R_{\sigma} F(x)=\sup _{k} \sup _{J \in \mathcal{J}^{k}} \sup _{t \in J}\left|\sum_{\substack{j \geq \sigma: \\ b_{J}-a_{J} \leq 2^{k-j} \\ 12}} \mathcal{A}_{t}^{j} G_{\sigma}^{j-k}(F)(x)\right|
$$


and, for $m \geq 0$

$$
S_{m, \sigma} F(x)=\sup _{k} \sup _{\substack{\mu: \\ \mu+m \geq \sigma}} \sup _{\substack{J \in \mathcal{J}_{\mu}^{k} \\ b_{J}-a_{J}>2^{k-\mu-m}}} \sup _{t \in J}\left|\mathcal{A}_{t}^{\mu+m} G_{\sigma}^{\mu+m-k}(F)(x)\right|
$$

Next let

$$
M_{\sigma} F(x)=\sup _{k} \sup _{\mu>\sigma} \sup _{J \in \mathcal{J}_{\mu}^{k}} \sup _{t \in J}\left|\sum_{\substack{\sigma<j<\mu \\ b_{J}-a_{J}>2^{k-j}}} \mathcal{A}_{t}^{j} G_{\sigma}^{j-k}(F)(x)\right| .
$$

Thus

$$
\mathfrak{M}_{\sigma} F(x) \leq R_{\sigma} F(x)+\sum_{m} S_{m, \sigma} F(x)+M_{\sigma} F(x)
$$

Finally, for $\ell>0$, let

$$
\mathcal{J}_{\mu}^{k, \ell}=\left\{J \in \mathcal{J}_{\mu}^{k}: b_{J}-a_{J} \geq 2^{k-\mu+\ell}\right\}
$$

and define

$$
M_{\ell, \sigma} F(x)=\sup _{k} \sup _{\mu>\ell+\sigma} \sup _{J \in \mathcal{J}_{\mu}^{k, \ell}} \sup _{t \in J}\left|\mathcal{A}_{t}^{\mu-\ell} G_{\sigma}^{\mu-\ell-k}(F)(x)\right|
$$

so that

$$
M_{\sigma} F(x) \leq \sum_{\ell>0} M_{\ell, \sigma} F(x)
$$

The claim in Theorem I will be a consequence of the following Propositions 4.2, 4.3, 4.4, in conjunction with Proposition 3.3.

The following result is essentially Proposition 4.1 applied to the set of 'endpoints', i.e. $\cup_{k} \mathcal{D}^{k}$.

Proposition 4.2. Suppose that $1 \leq p \leq 2$ and $E$ satisfies the regularity hypothesis $\left(\mathcal{R}_{p}\right)$, and let $\mathcal{D}^{k}$ be as in (1.9). Assume that $\left\{\omega_{n}^{-1}\right\} \in \ell^{p^{\prime}}$ with norm $\leq 1$. Then

$$
\left\|R_{\sigma} F\right\|_{L^{p}} \lesssim 2^{-\sigma(d-1)(1 / p-1 / 2)} \sup _{l}\left(\sum_{j \geq 0}\left[\omega_{j} N\left(\mathcal{D}^{j+l}, 2^{l}\right)^{1 / p} 2^{-j(d-1) / p^{\prime}}\right]^{p}\right)^{1 / p}\|F\|_{X^{p}}
$$

Proof. Using Hölder's inequality as above (with $\left\{\omega_{n}^{-1}\right\} \in \ell^{p^{\prime}}$ ) we may estimate

$$
R_{\sigma} F(x) \lesssim\left(\sum_{k} \sum_{j \geq \sigma} \omega_{j}^{p} \sup _{\substack{J: \\ b_{J}-a a_{J} \leq 2^{k-j}}} \sup _{t \in J}\left|\mathcal{A}_{t}^{j} G_{\sigma}^{j-k} F(x)\right|^{p}\right)^{1 / p} .
$$

Now if for fixed $j, k$ we let $\mathcal{E}=\cup_{J \in \mathcal{J}^{k}: b_{J}-a_{J} \leq 2^{k-j}} J$ then $N\left(\mathcal{E}, 2^{k-j}\right) \lesssim N\left(\mathcal{D}^{k}, 2^{k-j}\right)$. Hence by Lemma 2.2 (iii),

$$
\begin{aligned}
\left\|R_{\sigma} F\right\|_{L^{p}} & \lesssim\left(\sum_{k} \sum_{j} \omega_{j}^{p}\left\|\sup _{\substack{J \in \mathcal{J}^{k}: \\
b_{J}-a_{J} \leq 2^{k-j}}} \sup _{t \in J}\left|\mathcal{A}_{t}^{j} G_{\sigma}^{j-k}(F)\right|\right\|_{L^{p}}^{p}\right)^{1 / p} \\
& \lesssim 2^{-\sigma(d-1)(1 / p-1 / 2)}\left(\sum_{k} \sum_{j} \omega_{j}^{p} N\left(\mathcal{D}^{k}, 2^{k-j}\right) 2^{-j(d-1) p / p^{\prime}} \sum_{L(Q)=k-j+\sigma}|Q|^{1-p / 2}\left\|F_{Q}^{j-k}\right\|_{L^{2}}^{p}\right)^{1 / p}
\end{aligned}
$$

and from here on the proof goes exactly as for Proposition 4.1. 
Proposition 4.3. For $1 \leq p \leq 2$

$$
\left\|S_{m, \sigma} F\right\|_{L^{p}} \lesssim 2^{-m(d-1) / p^{\prime}} 2^{-\sigma(d-1)(1 / p-1 / 2)} \sup _{l}\left(\sum_{j \geq 0} N\left(E^{j+l}, 2^{l}\right) 2^{-j(d-1) p / p^{\prime}}\right)^{1 / p}\|F\|_{X^{p}}
$$

Proof. We have (using Lemma 2.2 (iii) for the sets $J \in \mathcal{J}_{\mu}^{k}$ and noting $N\left(J, 2^{k-\mu-m}\right) \approx \operatorname{card}(J)$ )

$$
\begin{aligned}
&\left\|S_{m, \sigma} F\right\|_{L^{p}} \lesssim\left\|\left(\sum_{k, \mu} \sum_{\substack{J \in \mathcal{J}_{\mu}^{k} \\
b_{J}-a_{J}>2^{k-\mu-m}}}\left[\sup _{t \in J}\left|\mathcal{A}_{t}^{\mu+m} \sum_{L(Q)=k-\mu-m+\sigma} F_{Q}^{\mu+m-k}\right|\right]^{p}\right)^{1 / p}\right\|_{L^{p}} \\
& \lesssim\left(\sum_{k, \mu} \sum_{J \in \mathcal{J}_{\mu}^{k}}\left[2^{-\sigma(d-1)(1 / p-1 / 2)} 2^{-(\mu+m)(d-1) / p^{\prime}} \operatorname{card}(J)^{1 / p}\right]^{p}\right. \\
&\left.\times\left(\sum_{L(Q)=k-\mu-m+\sigma}|Q|^{1-p / 2}\left\|F_{Q}^{\mu+m-k}\right\|_{L^{2}}^{p}\right)\right)^{1 / p} .
\end{aligned}
$$

Now by (1.10) the latter expression is estimated by $2^{-\sigma(d-1)(1 / p-1 / 2)} 2^{-m(d-1) / p^{\prime}}$ times the quantity

$$
\left(\sum_{k, \mu} N\left(E^{k}, 2^{k-\mu}\right) 2^{-\mu(d-1) p / p^{\prime}} \sum_{L(Q)=k-\mu-m+\sigma}|Q|^{1-p / 2}\left\|F_{Q}^{\mu+m-k}\right\|_{L^{2}}^{p}\right)^{1 / p}
$$

which is bounded by

$$
\sup _{\ell}\left\{\sum_{\mu} N\left(E^{\ell+\mu}, 2^{\ell}\right) 2^{-\mu(d-1) p / p^{\prime}}\right\}^{1 / p}\left(\sum_{l} \sum_{L(Q)=l-m+\sigma}|Q|^{1-p / 2}\left\|F_{Q}^{m-l}\right\|_{L^{2}}^{p}\right)^{1 / p} .
$$

This gives the claimed estimate.

Proposition 4.4. Suppose that

$$
\sup _{k} \sum_{j \geq 0} 2^{-j(d-1) p / p^{\prime}} N\left(E^{j+k}, 2^{k}\right) \leq C_{1}^{p} .
$$

Then for $1 \leq p \leq 2$ we have the inequality

$$
\left\|M_{\ell, \sigma} F\right\|_{L^{p}} \lesssim C_{1} 2^{-\sigma(d-1)(1 / p-1 / 2)} 2^{-\ell\left(1-d / p^{\prime}\right)}\|F\|_{X^{p}} .
$$

Proof. This is a small (but crucial) variation of the proof of Proposition 4.3. We have by Lemma 2.3 part (ii),

$$
\begin{aligned}
\left\|M_{\ell, \sigma} F\right\|_{L^{p}} \leq & \left(\sum_{k} \sum_{\mu>\ell+\sigma} \sum_{J \in \mathcal{J}_{\mu}^{k, \ell}}\left\|\sup _{t \in J}\left|\mathcal{A}_{t}^{\mu-\ell} G_{\sigma}^{\mu-\ell-k}(F)\right|\right\|_{L^{p}}^{p}\right)^{1 / p} \\
\lesssim & 2^{-\sigma(d-1)(1 / p-1 / 2)} \times \\
& \left(\sum_{k} \sum_{\mu>\ell} \sum_{J \in \mathcal{J}_{\mu}^{k, \ell}} \operatorname{card}(J) 2^{-(\mu-\ell)(d-1) p / p^{\prime}} 2^{-\ell} \sum_{Q: L(Q)=\sigma-\mu+\ell+k}|Q|^{1-p / 2}\left\|F_{Q}^{\mu-\ell-k}\right\|_{L^{2}}^{p}\right)^{1 / p}
\end{aligned}
$$

and this expression by $(1.10)$ is controlled by $2^{-\ell\left(1-d / p^{\prime}\right)} 2^{-\sigma(d-1)(1 / p-1 / 2)}$ times the expression

$$
\left(\sum_{n} \sum_{Q: L(Q)=\sigma+n+\ell}|Q|^{1-p / 2}\left\|F_{Q}^{-n-\ell}\right\|_{2}^{p} \sum_{\mu>\ell} N\left(E^{n+\mu}, 2^{n}\right) 2^{-\mu(d-1) p / p^{\prime}}\right)^{1 / p}
$$

which is

$$
\lesssim \sup _{n}\left(\sum_{\mu>\ell} N\left(E^{n+\mu}, 2^{n}\right) 2^{-\mu(d-1) p / p^{\prime}}\right)^{1 / p}\|F\|_{X^{p}}
$$

Thus (4.12) follows.

Proof of Theorem I. Immediate by Propositions 3.3, 4.2, 4.3 and 4.4. 


\section{Weak type $(p, p)$ estimates}

In this section we shall mostly assume that $p<d /(d-1)$ and

$$
\sup _{k} \sup _{j \geq 0} N\left(E^{k}, 2^{k-j}\right)^{1 / p} 2^{-j(d-1) / p^{\prime}} \leq C_{0} .
$$

Some statements however will extend to the limiting case $p=d /(d-1)$.

The proof of Theorem II follows from Proposition 3.3, (4.6), (4.9), Proposition 4.2 and estimates for the operators $S_{m, \sigma}$ and $M_{\ell, \sigma}$, stated in the following Propositions 5.1 and 5.2.

Proposition 5.1. Let $S_{m, \sigma} F$ be as in (4.4). Suppose that $1<p \leq d /(d-1)$ if $d=3$, and $1<p<$ $d /(d-1)=2$ if $d=2$, and assume that (5.1) is valid. Then there is $\varepsilon=\varepsilon(p)>0$ so that for all $\sigma, m \geq 0$

$$
\left\|S_{m, \sigma} F\right\|_{L^{p, \infty}} \lesssim 2^{-\varepsilon(\sigma+m)}\|F\|_{X^{p}} .
$$

Proposition 5.2. Let $M_{\ell, \sigma} F$ be as in (4.8). Suppose that $1<p<d /(d-1)$ and that (5.1) holds. Then there is $\varepsilon=\varepsilon(p)>0$ so that for $\sigma, \ell \geq 0$

$$
\left\|M_{\ell, \sigma} F\right\|_{L^{p, \infty}} \lesssim 2^{-\varepsilon(\sigma+\ell)}\|F\|_{X^{p}}
$$

Proof of Proposition 5.1. We have to show that for every $\alpha>0$

$$
\operatorname{meas}\left(\left\{x:\left|S_{m, \sigma} F(x)\right| \geq 3 \alpha\right\}\right) \lesssim 2^{-\varepsilon(m+\sigma) p} \alpha^{-p}\|F\|_{X^{p}}^{p} .
$$

Now fix $\alpha>0$ and let

$$
c_{Q}=|Q|^{1 / p-1 / 2}\left(\sum_{l: L(Q)+l \geq 0}\left\|F_{Q}^{l}\right\|_{L^{2}}^{2}\right)^{1 / 2}
$$

so that $\sum c_{Q}^{p}=\|F\|_{X^{p}}^{p}$. Fix a small $\varepsilon_{0}>0$ to be chosen later. We divide up the dyadic cubes into two families;

$$
\mathcal{G}=\left\{Q: c_{Q}^{p} \frac{1}{|Q|} \leq 2^{\varepsilon_{0}(\sigma+m) p} \alpha^{p}\right\},
$$

and complementary family $\Gamma$, so that $\{Q\}=\mathcal{G} \cup \Gamma$ and $\mathcal{G} \cap \Gamma=\emptyset$. Define

$$
\begin{aligned}
& \mathcal{G}(F)=\left\{F_{Q}^{l}\right\}_{\substack{L(Q)+l \geq 0 \\
Q \in \mathcal{G}}} \\
& \mathcal{B}(F)=\left\{F_{Q}^{l}\right\}_{\substack{L(Q)+l \geq 0 \\
Q \in \Gamma}}
\end{aligned}
$$

For $S_{m, \sigma} \mathcal{G}(F)$ we use a straightforward $L^{2}$ estimate. ¿From Lemma 2.2 (iii) (with $\left.\mathcal{E}=J \in \mathcal{J}_{\mu}^{k}\right),(1.10$ ) and (5.1) we deduce

$$
\begin{aligned}
& \left\|S_{m, \sigma} \mathcal{G}(F)\right\|_{L^{2}}^{2} \leq\left\|\left(\sum_{k} \sum_{\substack{\mu>0: \\
\mu+m \geq \sigma}} \sum_{\substack{J \in \mathcal{J}_{\mu}^{k} \\
b_{J}-a_{J}>2^{k-\mu-m}}} \sup _{t \in J}\left|\mathcal{A}_{t}^{\mu+m}\left[\sum_{\substack{Q \in \mathcal{G} \\
L(Q)=k-\mu-m+\sigma}} F_{Q}^{\mu+m-k}\right]\right|^{2}\right)^{1 / 2}\right\|_{L^{2}}^{2} \\
& \lesssim \sum_{k} \sum_{\substack{\mu>0: \\
\mu \geq \sigma-m}} N\left(E^{k}, 2^{k-\mu}\right) 2^{-(\mu+m)(d-1)} \sum_{\substack{Q \in \mathcal{G} \\
L(Q)=k-\mu-m+\sigma}}\left\|F_{Q}^{\mu+m-k}\right\|_{L^{2}}^{2} \\
& \lesssim 2^{-m(d-1)} \sum_{k} \sum_{\substack{\mu>0: \\
\mu \geq \sigma-m}} 2^{-\mu(d-1)(2-p)} \sum_{\substack{Q \in \mathcal{G} \\
L(Q)=k-\mu-m+\sigma}}\left\|F_{Q}^{\mu+m-k}\right\|_{L^{2}}^{2} .
\end{aligned}
$$


¿From (5.5) and (5.6) we have for $Q \in \mathcal{G}$

$$
\left\|F_{Q}^{\mu+m-k}\right\|_{L^{2}} \leq c_{Q} /|Q|^{1 / p-1 / 2} \leq 2^{\varepsilon_{0}(\sigma+m)}|Q|^{1 / 2} \alpha .
$$

By Čebyšev's inequality and (5.7), (5.8) we obtain

$$
\begin{aligned}
\operatorname{meas}(\{x & \left.\left.:\left|S_{m, \sigma} \mathcal{G}(F)(x)\right|>\alpha\right\}\right) \\
& \leq \alpha^{-2}\left\|S_{m, \sigma} \mathcal{G}(F)\right\|_{L^{2}}^{2} \\
& \lesssim \alpha^{-2} 2^{-m(d-1)} \sum_{k} \sum_{\substack{\mu>0: \\
\mu \geq \sigma-m}} 2^{-\mu(d-1)(2-p)} \sum_{\substack{Q \in \mathcal{G} \\
L(Q)=k-\mu-m+\sigma}}\left\|F_{Q}^{\mu+m-k}\right\|_{L^{2}}^{p}\left\|F_{Q}^{\mu+m-k}\right\|_{L^{2}}^{2-p} \\
& \lesssim \alpha^{-p} 2^{\varepsilon_{0} \sigma(2-p)} 2^{-m\left[(d-1)-\varepsilon_{0}(2-p)\right]} \sum_{k} \sum_{\substack{\mu>0: \\
\mu \geq \sigma-m}} 2^{-\mu(d-1)(2-p)} \sum_{Q \in \mathcal{G}}|Q|^{1-p / 2}\left\|F_{Q}^{\sigma-L(Q)}\right\|_{L^{2}}^{p} \\
& \lesssim \alpha^{-p} 2^{-\varepsilon \sigma} 2^{-\varepsilon m}\|F\|_{X^{p}}^{p}
\end{aligned}
$$

for some $\varepsilon>0$ if $\varepsilon_{0}>0$ is small enough.

We now concentrate on the family $\Gamma$ of dyadic cubes which do not belong to $\mathcal{G}$. Define

$$
A(Q, \tau) \equiv A_{\alpha, \sigma, m}(Q, \tau):=2^{(\sigma+m) \varepsilon_{0} p} \alpha^{p} 2^{\tau(d-1) p} 2^{L(Q)\left[\frac{1}{p}-\frac{d-1}{p^{\prime}}\right] p}
$$

note that $\tau \mapsto A(Q, \tau)$ defines an increasing unbounded sequence for $\tau \geq L(Q)$ and in particular

$$
A(Q, L(Q))=2^{(\sigma+m) \varepsilon_{0} p} \alpha^{p}|Q|
$$

so that for every $Q \in \Gamma, c_{Q}^{p}>A(Q, L(Q))$.

Definition. For every $Q \in \Gamma$ we define $\tau(Q)$ to be the smallest integer $\tau>L(Q)$ so that $A(Q, \tau) \geq c_{Q}^{p}$.

For each $Q \in \Gamma$ we then define $k(Q, \gamma)=(L(Q)+\gamma \tau(Q)) /(\gamma+1)$ and

$$
W(Q)=\bigcup_{k(Q, \gamma)<k \leq \tau(Q)} \bigcup_{I \in \mathfrak{I}_{L(Q)}\left(E^{k}\right)}\left\{x \in \mathbb{R}^{d}:|| x-x_{Q}\left|-r_{I}\right| \leq 2^{L(Q)+4} 2^{(\tau(Q)-k) \gamma}\right\}
$$

where $\gamma<(d-1) p$ and note that

$$
\begin{aligned}
\operatorname{meas}(W(Q)) & \lesssim \sum_{k \leq \tau(Q)} N\left(E^{k}, 2^{k-(k-L(Q))}\right) 2^{L(Q)+k(d-1)} 2^{(\tau(Q)-k) \gamma} \\
& \lesssim \sum_{k \leq \tau(Q)} 2^{(\tau(Q)-k) \gamma} 2^{(k-L(Q)) \frac{d-1}{p^{\prime}} p} 2^{L(Q)+k(d-1)} \\
& \lesssim 2^{\tau(Q)(d-1) p} 2^{L(Q)\left[\frac{1}{p}-\frac{d-1}{p^{\prime}}\right] p} .
\end{aligned}
$$

Let

$$
\mathcal{W}=\bigcup_{Q \in \Gamma}\left(\left\{x \in \mathbb{R}^{d}:\left|x-x_{Q}\right| \leq 2^{k(Q, \gamma)+4}\right\} \cup W(Q)\right) .
$$

By (5.10), (5.13) and the definition of $\tau(Q)$

$$
\begin{aligned}
\operatorname{meas}(\mathcal{W}) \lesssim \sum_{Q \in \Gamma}\left[2^{k(Q, \gamma) d}\right. & +\operatorname{meas}(W(Q))] \lesssim 2^{\tau(Q)(d-1) p} 2^{L(Q)\left[\frac{1}{p}-\frac{d-1}{p^{\prime}}\right] p} \\
& \lesssim \sum_{Q \in \Gamma} 2^{-(\sigma+m) \varepsilon_{0} p} \alpha^{-p} A(Q, \tau(Q)) \\
& \lesssim 2^{-(\sigma+m) \varepsilon_{0} p} \alpha^{-p} \sum_{Q \in \Gamma} c_{Q}^{p} \lesssim 2^{-(\sigma+m) \varepsilon_{0} p} \alpha^{-p}\|F\|_{X^{p}}^{p}
\end{aligned}
$$


It remains to be shown that

$$
\operatorname{meas}\left(\left\{x \notin \mathcal{W}: S_{m, \sigma}(\mathcal{B}(F))>2 \alpha\right\}\right) \lesssim 2^{-(\sigma+m) \varepsilon_{0} p} \alpha^{-p}\|F\|_{X^{p}}^{p}
$$

We split $S_{m, \sigma}(\mathcal{B}(F))=\sum_{s=-\infty}^{\infty} I_{s}$ where

$$
I_{s}=\sup _{k} \sup _{\mu+m-\sigma \geq \max \{s, 0\}} \sup _{\substack{J \in \mathcal{J}_{\mu}^{k} \\ b_{J}-a_{J} \geq 2^{k-\mu-m}}} \sup _{t \in J}\left|\mathcal{A}_{t}^{\mu+m}\left[\sum_{\substack{Q \in \Gamma \\ L(Q)=k-\mu-m+\sigma \\ \tau(Q)=k-s}} F_{Q}^{\mu+m-k}\right]\right| .
$$

We shall prove

$$
\left\|I_{s}\right\|_{L^{2}}^{2} \lesssim 2^{-s(d-1)(2-p)} 2^{-\sigma(2-p)\left(d-1-\varepsilon_{0}\right)} 2^{-m\left[(d-1)(p-1)-\varepsilon_{0}(2-p)\right]} \alpha^{2-p}\|F\|_{X^{p}}^{p}, \quad s \geq 0,
$$

and

$$
\left\|I_{s}\right\|_{L^{p}\left(\mathbb{R}^{d} \backslash \mathcal{W}\right)}^{p} \leq C_{M^{2}} 2^{-M(\sigma+\gamma|s|)(2-p)} 2^{-m(d-1)(p-1)}\|F\|_{X^{p}}^{p}, \quad s \leq 0 .
$$

Note that for $\varepsilon_{0}>0$ small enough inequalities (5.15) and (5.16) imply (5.14) since

$$
\begin{aligned}
\operatorname{meas}(\{x \notin \mathcal{W}: & \left.\left.S_{m, \sigma}(\mathcal{B}(F))>2 \alpha\right\}\right) \\
& \leq \alpha^{-2}\left\|\sum_{s \geq 0} I_{s}\right\|_{L^{2}}^{2}+\alpha^{-p}\left\|\sum_{s<0} I_{s}\right\|_{L^{p}\left(\mathbb{R}^{d} \backslash \mathcal{W}\right)}^{p} \\
& \lesssim 2^{-\varepsilon(\sigma+m) p} \alpha^{-p}\|F\|_{X^{p}}^{p}
\end{aligned}
$$

for suitable $\varepsilon=\varepsilon(p)>0$.

Proof of (5.15). We use Lemma 2.2 (iii) for $\mathcal{E}=J \in \mathcal{J}_{\mu}^{k},(1.10)$ and (5.1) to obtain

$$
\begin{aligned}
& \left\|I_{s}\right\|_{L^{2}}^{2} \lesssim \sum_{k, \mu, J}\left\|\sup _{t \in J} \mathcal{A}_{t}^{\mu+m}\left[\sum_{\substack{Q \in \Gamma \\
\tau(Q)=k-s \\
L(Q)=k-\mu-m+\sigma}} F_{Q}^{\mu+m-k}\right]\right\|_{L^{2}}^{2} \\
& \lesssim \sum_{k, \mu, J} \operatorname{card}(J) 2^{-(\mu+m)(d-1)} \sum_{\substack{Q \in \Gamma \\
\tau(Q)=k-s \\
L(Q)=k-\mu-m+\sigma}}\left\|F_{Q}^{\mu+m-k}\right\|_{L^{2}}^{2} \\
& \lesssim 2^{-m(d-1)} \sum_{k, \mu} 2^{-\mu(d-1)(2-p)} \sum_{\substack{Q \in \Gamma \\
\tau(Q)=k-s \\
L(Q)=k-\mu-m+\sigma}}\left\|F_{Q}^{\mu+m-k}\right\|_{L^{2}}^{2} .
\end{aligned}
$$

As $k=\tau(Q)+s$ and $\mu=\tau(Q)-L(Q)+s+\sigma-m$ this inequality can be rewritten as

$$
\left\|I_{s}\right\|_{L^{2}}^{2} \lesssim 2^{-m(d-1)} \sum_{Q \in \Gamma} 2^{(\tau(Q)-L(Q)+s+\sigma-m)(d-1)(p-2)}\left\|F_{Q}^{\sigma-L(Q)}\right\|_{L^{2}}^{2}
$$

Now we use that for $Q \in \Gamma$

$$
\begin{aligned}
& \left\|F_{Q}^{\sigma-L(Q)}\right\|_{L^{2}}^{2-p} \leq\left(c_{Q}|Q|^{1 / p-1 / 2}\right)^{2-p} \leq\left(2^{-d(1 / p-1 / 2) L(Q)} A(Q, \tau(Q))\right)^{2-p} \\
& \lesssim\left[\alpha 2^{\varepsilon_{0}(\sigma+m)} 2^{(d-1) \tau(Q)} 2^{L(Q)\left(\frac{1}{p}-\frac{d-1}{p^{\prime}}-d\left(\frac{1}{p}-\frac{1}{2}\right)\right)}\right]^{2-p} \\
& 17
\end{aligned}
$$


and combine this with (5.18) to obtain after a little algebra

$$
\left\|I_{s}\right\|_{L^{2}}^{2} \lesssim 2^{m\left((d-1)(1-p)+\varepsilon_{0}(2-p)\right)} 2^{-\sigma\left(d-1-\varepsilon_{0}\right)(2-p)} 2^{-s(d-1)(2-p)} \alpha^{2-p} \sum_{Q}|Q|^{1-p / 2}\left\|F_{Q}\right\|_{L^{2}}^{p}
$$

which is the desired bound.

Proof of (5.16). We use the estimate away from the exceptional set in Lemma 2.2 (iv), with $\eta=2^{|s| \gamma}$ $(\gamma<(d-1) p)$ and $s=k-\tau(Q)$. Then

$$
\begin{aligned}
\left\|I_{s}\right\|_{L^{p}\left(\mathbb{R}^{d} \backslash \mathcal{W}\right)}^{p} & \lesssim \sum_{k, \mu}\left\|\sup _{t \in J} \mathcal{A}_{t}^{\mu+m}\left[\sum_{\substack{\tau(Q)=k-s \\
L(Q)=k-\mu-m+\sigma}} F_{Q}^{\mu+m-k}\right]\right\|_{L^{p}\left(\mathbb{R}^{d} \backslash \mathcal{W}\right)}^{p} \\
& \lesssim \sum_{k, \mu} N\left(E^{k}, 2^{k-\mu}\right) 2^{-(\mu+m)(d-1) p / p^{\prime}} 2^{-(\sigma+\gamma|s|) M(2-p)} \sum_{\substack{\tau(Q)=k-s \\
L(Q)=k-\mu-m+\sigma}}|Q|^{1-p / 2}\left\|F_{Q}^{\mu+m-k}\right\|_{L^{2}}^{p} \\
& \lesssim 2^{-m(d-1) p / p^{\prime}} 2^{-\sigma M(2-p)} 2^{-|s| \gamma(2-p)}\|F\|_{X^{p}}^{p} . \quad \square
\end{aligned}
$$

\section{Proof of Proposition 5.2.}

This is similar to the proof of Proposition 5.1. We have to show that for every $\alpha>0$

$$
\operatorname{meas}\left(\left\{x:\left|M_{\ell, \sigma} F(x)\right| \geq 3 \alpha\right\}\right) \lesssim 2^{-\varepsilon(\ell+\sigma) p} \alpha^{-p}\|F\|_{X^{p}}^{p} .
$$

We indicate the changes in the proof of Proposition 5.1. Of course we systematically replace $S_{m, \sigma}$ by $M_{\ell, \sigma}$. The definition (5.6) is the same except that $2^{\varepsilon_{0} m p}$ has to be replaced by $2^{\varepsilon_{0} \ell p}$; then the arguments up to (5.9) are similar; we have to use Lemma 2.3 (ii) instead of Lemma 2.2 (iii). Similarly the definition (5.10) is changed to

$$
A(Q, \tau) \equiv A_{\alpha, \sigma, \ell}(Q, \tau):=2^{(\sigma+\ell) \varepsilon_{0} p} \alpha^{p} 2^{\tau(d-1) p} 2^{L(Q)\left[\frac{1}{p}-\frac{d-1}{p^{\prime}}\right] p}
$$

and the further arguments up to (5.14) have obvious analogues. In the definition of $A(Q, \tau)$ we shall need to take $\varepsilon_{0}$ so that $\varepsilon_{0}(2-p)<1-(d-1)(p-1)$ which is possible since $p<d /(d-1)$.

We then split $M_{\ell, \sigma}(\mathcal{B}(F))=\sum I I_{s}$ where

$$
\begin{array}{r}
I I_{s}=\sup _{k} \sup _{\mu \geq \ell+\sigma} \sup _{J \in \mathcal{J}_{\mu}^{k, \ell}} \sup _{t \in J}\left|\mathcal{A}_{t}^{\mu-\ell} G_{\sigma, s}^{\mu-\ell-k}(F)\right| \\
\text { and } G_{\sigma, s}^{\mu-\ell-k} F:=\sum_{\substack{Q: L(Q)=k-\mu+\ell+\sigma \\
\tau(Q)=k-s}} F_{Q}^{\mu-\ell-k} .
\end{array}
$$

The inequalities (5.15) and (5.16) are replaced by

$$
\left\|I I_{s}\right\|_{L^{2}}^{2} \lesssim 2^{-s(d-1)(2-p)} 2^{-\sigma(2-p)\left(d-1-\varepsilon_{0}\right)} 2^{-\ell\left[(1-(d-1)(p-1))-\varepsilon_{0}(2-p)\right]} \alpha^{2-p}\|F\|_{X^{p}}^{p}, \quad s \geq 0,
$$

and

$$
\left\|I I_{s}\right\|_{L^{p}\left(\mathbb{R}^{d} \backslash \mathcal{W}\right)}^{p} \lesssim 2^{-\ell\left(1-\frac{d}{p^{\prime}}\right) p} 2^{-s(d-1)(1-p / 2)} 2^{-(\sigma+|s| \gamma) M p} \alpha^{2-p}\|F\|_{X^{p}}^{p}, \quad s \leq 0,
$$

from which we can as before conclude the assertion of the proposition. 
Proof of (5.21) and (5.22). We prove (5.21) and use Lemma 2.3 to estimate

$$
\begin{aligned}
\left\|I I_{s}\right\|_{L^{2}}^{2} & \leq \sum_{k} \sum_{\mu \geq \ell+\sigma} \sum_{J \in \mathcal{J}_{\mu}^{k, \ell}}\left\|\sup _{t \in J} \mid \mathcal{A}_{t}^{\mu-\ell} G_{\sigma, s}^{\mu-\ell-k}(F)\right\|_{L^{2}}^{2} \\
& \leq \sum_{k} \sum_{\mu \geq \ell+\sigma} \sum_{J \in \mathcal{J}_{\mu}^{k, \ell}} \operatorname{card}(J) 2^{-(\mu-\ell)(d-2)} 2^{-\mu} \sum_{\substack{Q \in \Gamma \\
L(Q)=k-\mu+\ell+\sigma \\
\tau(Q)=k-s}}\left\|F_{Q}^{\mu-\ell-k}\right\|_{L^{2}}^{2}
\end{aligned}
$$

Now $\sum_{J \in \mathcal{J}_{\mu}^{k, \ell}} \operatorname{card} J \lesssim N\left(E^{k}, 2^{k-\mu}\right) \lesssim 2^{\mu(d-1)(p-1)}$ by assumption $(1.10)$ and $\left(\mathcal{C}_{p, \infty}\right)$. We also observe that $\mu=\tau(Q)-L(Q)+s+\sigma+\ell$ in the above sum and thus we obtain

$$
\begin{aligned}
\left\|I I_{s}\right\|_{L^{2}}^{2} & \lesssim 2^{\ell(d-2)} \sum_{k} \sum_{\mu \geq \ell+\sigma} 2^{\mu(d-1)(p-2)} \sum_{\substack{Q \in \Gamma \\
L(Q)=k-\mu+\ell+\sigma \\
\tau(Q)=k-s}}\left\|F_{Q}^{\mu-\ell-k}\right\|_{L^{2}}^{2} \\
& \lesssim 2^{\ell(d-2)} \sum_{\substack{Q \in \Gamma \\
\tau(Q)-L(Q) \geq-s}}\left\|F_{Q}^{\sigma-L(Q)}\right\|_{L^{2}}^{2} 2^{(\tau(Q)-L(Q)+s+\sigma+\ell)(d-1)(p-2)} .
\end{aligned}
$$

Now as before $\left\|F_{Q}^{\sigma-L(Q)}\right\|_{L^{2}}^{2-p} \lesssim\left[\alpha 2^{\varepsilon_{0}(\sigma+\ell)} 2^{(d-1) \tau(Q)} 2^{L(Q)\left(\frac{1}{p}-\frac{d-1}{p^{\prime}}-d\left(\frac{1}{p}-\frac{1}{2}\right)\right)}\right]^{2-p}$ and after doing the algebra we arrive at

$$
\left\|I I_{s}\right\|_{2}^{2} \lesssim \alpha^{2-p} 2^{\ell\left((d-1) p-d+\varepsilon_{0}(2-p)\right)} 2^{-\sigma\left(d-1-\varepsilon_{0}\right)(2-p)} 2^{-s(d-1)(2-p)} \sum_{Q \in \Gamma}|Q|^{1-p / 2}\left\|F_{Q}^{\sigma-L(Q)}\right\|_{L^{2}}^{p}
$$

which is what we were aiming for.

Similarly, the proof of (5.22) is analogous to the proof of (5.16).

\section{6. $L^{p}$ estimates in the limiting case}

We assume throughout this section that that the regularity condition $\left(\widetilde{\mathcal{R}}_{p_{d}}\right), p_{d}=d /(d-1)$, is satisfied. We first give a reformulation of the Carleson-measure condition.

Lemma 6.1. Suppose that the Carleson measure condition $\left(\widetilde{\mathcal{C}}_{p_{d}}\right)$ holds. Then the measure

$$
\sum_{k \in \mathbb{Z}} \sum_{\mu \geq 0} \delta_{k, \mu} \sum_{J \in \mathcal{J}_{\mu}^{k}} \operatorname{card}(J) 2^{-\mu}(1+\mu)^{\frac{d}{d-1}}
$$

is also a Carleson measure.

Proof. We first observe that

$$
N\left(E^{k}, 2^{k-j}\right) 2^{k-j} \approx\left|\left\{t \in\left[2^{k}, 2^{k+1}\right): \operatorname{dist}\left(t, E^{k}\right) \leq 2^{k-j}\right\}\right|
$$

and thus

$$
N\left(E^{k}, 2^{k-j}\right) 2^{-j} \leq C N\left(E^{k}, 2^{k-j^{\prime}}\right) 2^{-j^{\prime}} \text { if } j^{\prime} \leq j .
$$


Let $I$ be an interval of length $>1$ and $I^{*}$ the interval with same midpoint and double length. Then

$$
\begin{aligned}
& \sum_{(k, \mu) \in T(I)} \sum_{J \in \mathcal{J}_{\mu}^{k}} \operatorname{card}(J) 2^{-\mu}(1+\mu)^{d /(d-1)} \\
\lesssim & \sum_{k \in I} \sum_{s=0}^{1+\log _{2}|I|} 2^{s d /(d-1)} \sum_{2^{s-1} \leq \mu<2^{s}} 2^{-\mu} \sum_{J \in \mathcal{J}_{\mu}^{k}} \operatorname{card}(J) \\
\lesssim & \sum_{k \in I} \sum_{s=0}^{1+\log _{2}|I|} 2^{s d /(d-1)} N\left(E^{k}, 2^{k-2^{s-1}}\right) 2^{-2^{s-1}} \\
\lesssim & \sum_{k \in I} \sum_{s=0}^{1+\log _{2}|I|} \sum_{2^{s-2} \leq \mu<2^{s-1}} N\left(E^{k}, 2^{k-\mu}\right) 2^{-\mu}(1+\mu)^{1 /(d-1)} \\
\lesssim & \sum_{(k, \mu) \in T\left(I^{*}\right)} N\left(E^{k}, 2^{k-\mu}\right) 2^{-\mu}(1+\mu)^{1 /(d-1)} .
\end{aligned}
$$

Here we have used the regularity assumption (1.11) for the second inequality and (6.1) for the third inequality.

The following is an even more elementary observation.

Lemma 6.2. Suppose that the Carleson measure condition $\left(\widetilde{\mathcal{C}}_{p_{d}}\right)$ holds. Then

$$
\sup _{\nu} \sum_{\mu} \sum_{J \in \mathcal{J}_{\mu}^{\mu+\nu}} \operatorname{card}(J) 2^{-\mu} \leq C .
$$

Proof. Let $I_{s}(r)=\left\{x:|x-r| \leq 2^{s}\right\}$. Then

$$
\begin{aligned}
\sum_{\mu} \sum_{J \in \mathcal{J}_{\mu}^{\mu+r}} \operatorname{card}(J) 2^{-\mu} & \lesssim \sum_{s=0}^{\infty} 2^{-s d /(d-1)} \sum_{0 \leq \mu \leq 2^{s}} \sum_{J \in \mathcal{J}_{\mu}^{\mu+r}} \operatorname{card}(J) 2^{-\mu}(1+\mu)^{d /(d-1)} \\
& \lesssim \sum_{s=0}^{\infty} 2^{-s /(d-1)} \frac{1}{\left|I_{s}(r)\right|} \sum_{(k, \mu) \in T\left(I_{s}(r)\right)} \sum_{J \in \mathcal{J}_{\mu}^{k}} \operatorname{card}(J) 2^{-\mu}(1+\mu)^{d /(d-1)}
\end{aligned}
$$

and the last expression is bounded by Lemma 6.1 .

The following Carleson-measure estimate is a standard consequence of the $L^{p}$ boundedness of the Hardy-Littlewood maximal operator, for the proof see [14, ch. II.2].

Lemma 6.3. Suppose the doubly indexed nonnegative sequence $\left\{\omega_{k, \mu},(k, \mu) \in \mathbb{Z} \times \mathbb{Z}^{+}\right\}$satisfies

$$
\sup _{|I| \geq 1} \frac{1}{|I|} \sum_{(k, \mu) \in T(I)} \omega_{k, \mu} \leq A^{p}
$$

i.e. $\sum \omega_{k, \mu} \delta_{k, \mu}$ is a Carleson measure. Then for $\left\{a_{k}\right\} \in \ell^{p}, p>1$

$$
\left(\sum_{k, \mu} \omega_{k, \mu}\left[\frac{1}{1+\mu} \sum_{|j| \leq \mu}\left|a_{k+j}\right|\right]^{p}\right)^{1 / p} \leq C_{p} A\left(\sum_{k}\left|a_{k}\right|^{p}\right)^{1 / p} .
$$

We now turn to the 
$\boldsymbol{L}^{\mathbf{2}}$ estimates in two dimensions. We are concerned with the $L^{2}\left(\mathbb{R}^{2}\right)$ estimates in Theorem III. The claim is a consequence of the following estimates:

$$
\left\|\sup _{k, \mu} \sup _{J \in \mathcal{J}_{\mu}^{k}} \sup _{t \in J}\left|\sum_{\substack{j \leq \mu \\ b_{J}-a_{J}>2^{k-j}}} \mathcal{A}_{t}^{j} f\right|\right\|_{L^{2}} \lesssim\|f\|_{L^{2}}
$$

and, for $m \geq 0$,

$$
\left\|\sup _{k, \mu} \sup _{\substack{J \in \mathcal{J}_{\mu}^{k} \\ b_{J}-a_{j}>2^{k-\mu-m}}} \sup _{t \in J}\left|\mathcal{A}_{t}^{\mu+m} f\right|\right\|_{L^{2}} \lesssim 2^{-m / 2}\|f\|_{L^{2}}
$$

and finally

$$
\left\|\sup _{k} \sup _{J \in \mathcal{J}^{k}} \sup _{t \in J}\left|\sum_{\substack{j: \\ b_{J}-a_{J} \leq 2^{k-j}}} \mathcal{A}_{t}^{j} f\right|\right\|_{L^{2}} \lesssim\|f\|_{L^{2} .}
$$

To prove (6.3) we use Lemma 2.3 to see that the left side is dominated by

$$
\begin{aligned}
& \left(\sum_{k, \mu} \sum_{J \in \mathcal{J}_{\mu}^{k}}\left[\sum_{\substack{j \leq \mu \\
b_{J}-a_{J}>2^{k-j}}}\left\|\sup _{t \in J}\left|\mathcal{A}_{t}^{j} f\right|\right\|_{L^{2}}\right]^{2}\right)^{1 / 2} \\
& \lesssim\left(\sum_{k, \mu} \sum_{J \in \mathcal{J}_{\mu}^{k}} \operatorname{card}(J) 2^{-\mu}(1+\mu)^{2}\left[\frac{1}{1+\mu} \sum_{j \leq \mu}\left\|P^{j-k} f\right\|_{L^{2}}\right]^{2}\right)^{1 / 2}
\end{aligned}
$$

and by Lemma 6.3 and 6.1 the last expression is controlled by

$$
\left(\sum_{k \in \mathbb{Z}}\left\|P^{k} f\right\|_{L^{2}}^{2}\right)^{1 / 2} \lesssim\|f\|_{L^{2}}
$$

Concerning (6.4) we use Lemma 2.2 and bound the left side by

$$
\begin{aligned}
& \left(\sum_{k, \mu} \sum_{\substack{J \in \mathcal{J}_{\mu}^{k} \\
b_{J}-a_{J}>2^{k-j}}}\left\|\sup _{t \in J}\left|\mathcal{A}_{t}^{\mu+m} f\right|\right\|_{L^{2}}^{2}\right)^{1 / 2} \\
& \lesssim\left(\sum_{k, \mu} \sum_{J \in \mathcal{J}_{\mu}^{k}} N\left(J, 2^{k-m-\mu}\right) 2^{-(\mu+m)}\left\|P^{\mu+m-k} f\right\|_{L^{2}}^{2}\right)^{1 / 2} \\
& \lesssim 2^{-m / 2} \sup _{l \in \mathbb{Z}}\left(\sum_{\mu} \sum_{J \in \mathcal{J}_{\mu}^{\mu+m-l}} \operatorname{card}(J) 2^{-\mu}\right)^{1 / 2}\left(\sum_{k}\left\|P^{k} f\right\|_{L^{2}}^{2}\right)^{1 / 2}
\end{aligned}
$$

and by Lemma 6.2 the last expression is $\lesssim 2^{-m / 2}\|f\|_{L^{2}}$.

Finally (6.5) holds in view of the assumption (1.9); cf. the argument in the proof of Proposition 4.1. We shall not repeat the details. 
$X^{p}$ estimates and the proof of Theorem III. We use a similar decomposition as in $\S 4$ however instead of considering the maximal operators $M_{\ell, \sigma}$ we shall not decompose in $\ell$ and work with $M_{\sigma}$ in (4.5) directly. We shall prove

$$
\left\|M_{\sigma} F\right\|_{L^{p_{d}}} \lesssim 2^{-\sigma(d-1)\left(1 / p_{d}-1 / 2\right)}\|F\|_{X^{p_{d}}}
$$

This together with already proved estimates in $\S 4$ implies the statement of Theorem III.

We argue as before and set $a_{l}=\left(\sum_{L(Q)=\sigma-l}|Q|^{1-p_{d} / 2}\left\|F_{Q}^{l}\right\|_{L^{2}}^{p_{d}}\right)^{1 / p_{d}}$. Using Lemma 2.3 (ii) we get

$$
\begin{aligned}
\left\|M_{\sigma} F\right\|_{L^{p_{d}}} & \lesssim\left(\sum_{k} \sum_{\mu} \sum_{J \in \mathcal{J}_{\mu}^{k}}\left[\sum_{\substack{j \leq \mu \\
b_{J}-a_{J}>2^{k-j}}}\left\|\sup _{t \in J}\left|\mathcal{A}_{t}^{j} G_{\sigma}^{j-k}(F)\right|\right\|_{L^{p_{d}}}\right]^{p_{d}}\right)^{1 / p_{d}} \\
& \lesssim\left(\sum_{k} \sum_{\mu} \sum_{J \in \mathcal{J}_{\mu}^{k}}\left[\sum_{10<j \leq \mu} 2^{-\sigma(d-1)\left(1 / p_{d}-1 / 2\right)} \operatorname{card}(J)^{1 / p_{d}} 2^{-\mu / p_{d}} a_{j-k}\right]^{p_{d}}\right)^{1 / p_{d}} \\
& \lesssim 2^{-\sigma(d-1)\left(1 / p_{d}-1 / 2\right)}\left(\sum_{k} \sum_{\mu} \sum_{J \in \mathcal{J}_{\mu}^{k}} \operatorname{card}(J) 2^{-\mu} \mu^{p_{d}}\left(\frac{1}{\mu+1} \sum_{0 \leq j \leq \mu} a_{j-k}\right)^{p_{d}}\right)^{1 / p_{d}}
\end{aligned}
$$

By condition $\left(\widetilde{\mathcal{C}_{p_{d}}}\right)$ and Lemma 6.3 and Lemma 6.1 we obtain $(6.6)$.

\section{Weak type $(p, p)$ estimates in the limiting case}

Throughout this section we shall assume that $d \geq 3$ and that the regularity assumption and condition (1.14) hold; thus

$$
\sup _{k} 2^{-n} N\left(E^{k}, 2^{k-n}\right) \leq C\left(n^{\frac{1}{d-1}} \log n\right)^{-1}
$$

uniformly in $n \geq 10$. We follow the proof of Theorem II in $\S 5$, using the same decompositions except we do not decompose $M_{\sigma}$ in (4.5) further as in the proof of Theorem III. We recall that Proposition 5.1 remains valid for the limiting case $p=p_{d}$ if $d \geq 3$, under the weaker condition $\left(\mathcal{C}_{p_{d}, \infty}\right)$. Therefore the claim in Theorem IV will be a consequence of

Proposition 7.1. Let $M_{\sigma} F$ be as in (4.5). Suppose (7.1) holds. Then there is an $\varepsilon>0$ so that for all $\sigma, \alpha \geq 0$,

$$
\operatorname{meas}\left(\left\{x: M_{\sigma} F(x)>3 \alpha\right\}\right) \lesssim 2^{-\varepsilon \sigma p_{d}} \alpha^{-p_{d}}\|F\|_{X^{p_{d}}}^{p_{d}} .
$$

Proof. As in $\S 5$ we fix $\varepsilon_{0}>0$ and define $\mathcal{G}, \Gamma, A(Q, \tau), \mathcal{G}(F), \mathcal{B}(F)$ and $\mathcal{W}$ as before except we replace $2^{\varepsilon_{0}(\sigma+m) p}$ with $2^{\varepsilon_{0} \sigma p_{d}}$. In particular we have now for $\tau \geq L(Q)$

$$
A(Q, \tau)^{1 / p_{d}}=2^{\sigma \varepsilon_{0}} \alpha 2^{\tau(d-1)} .
$$

We shall have to take $\varepsilon_{0}$ so that $0<\varepsilon_{0}\left(2-p_{d}\right)<d-2$.

For $M_{\sigma} \mathcal{G}(F)$ we use an $L^{2}$ estimate. From Lemma 2.3 (ii) and the regularity assumption (1.11), we deduce

$$
\begin{aligned}
\left\|M_{\sigma} \mathcal{G}(F)\right\|_{L^{2}}^{2} & \leq \sum_{k} \sum_{\mu \geq \sigma} \sum_{J \in \mathcal{J}_{\mu}^{k}}\left(\sum_{\sigma \leq j \leq \mu}\left\|\sup _{t \in J} A_{t}^{j}\left(\sum_{\substack{Q \in \mathcal{G} \\
L(Q)=k-j+\sigma}} F_{Q}^{j-k}\right)\right\|_{L^{2}}\right)^{2} \\
& \lesssim \sum_{k} \sum_{\mu \geq \sigma} 2^{-\mu} \sum_{J \in \mathcal{J}_{\mu}^{k}} \operatorname{card}(J)\left(\sum_{\sigma \leq j \leq \mu} 2^{-j(d-2) / 2}\left(\sum_{\substack{Q \in \mathcal{G} \\
L(Q)=k-j+\sigma}}\left\|F_{Q}^{j-k}\right\|_{L^{2}}^{2}\right)^{1 / 2}\right)^{2} \\
& \left.\lesssim \sup _{k} 2^{-\sigma} N\left(E^{k}, 2^{k-\sigma}\right)\right]\left(\sum_{\sigma \leq j} 2^{-j(d-2) / 2}\left(\sum_{Q \in \mathcal{G}}\left\|F_{Q}^{\sigma-L(Q)}\right\|_{L^{2}}^{2}\right)^{1 / 2}\right)^{2} \\
& \lesssim 2^{\sigma(d-2)} \sum_{Q \in \mathcal{G}}\left\|F_{Q}^{\sigma-L(Q)}\right\|_{L^{2}}^{2} .
\end{aligned}
$$


For $Q \in \mathcal{G}$ we have

$$
\left\|F_{Q}^{\sigma-L(Q)}\right\|_{L^{2}} \leq c_{Q} /|Q|^{1 / p_{d}-1 / 2} \leq 2^{\varepsilon_{0} \sigma\left(2-p_{d}\right)}|Q|^{1 / 2} \alpha
$$

and therefore by Čebyšev's inequality and (7.3),

$$
\begin{aligned}
& \operatorname{meas}\left(\left\{M_{\sigma}(F)>\alpha\right\}\right) \\
& \quad \leq \alpha^{-2}\left\|M_{\sigma}(F)\right\|_{L^{2}}^{2} \\
& \lesssim 2^{-\sigma\left[(d-2)-\varepsilon_{0}\left(2-p_{d}\right)\right]} \alpha^{-p_{d}} \sum_{Q}|Q|^{1-p_{d} / 2}\left\|F_{Q}^{\sigma-L(Q)}\right\|_{L^{2}}^{p_{d}} \\
& \lesssim 2^{-\varepsilon \sigma} \alpha^{-p_{d}}\|F\|_{X^{p_{d}}}^{p_{2}}
\end{aligned}
$$

for some $\varepsilon>0$.

Furthermore the estimate for the measure of the exceptional set $\mathcal{W}$ in $\S 5$ is still valid. Therefore it remains to be shown that

$$
\operatorname{meas}\left(\left\{x \notin \mathcal{W}: M_{\sigma} \mathcal{B}(F)(x)>3 \alpha\right\}\right) \lesssim 2^{-\varepsilon \sigma} \alpha^{-p_{d}}\|F\|_{X^{p_{d}}}^{p_{d}}
$$

for some $\varepsilon>0$. We may estimate

$$
M_{\sigma} \mathcal{B}(F) \leq I I I+\sum_{s=0}^{\infty} I V_{s}+\sum_{s=-\infty}^{-1} V_{s}
$$

where

$$
\begin{aligned}
& I I I=\sup _{k} \sup _{\mu \geq \sigma} \sup _{J \in \mathcal{J}_{\mu}^{k}} \sup _{t \in J}\left|\sum_{\substack{\sigma \leq j \leq \mu \\
b_{J}-a_{J} \geq 2^{k-j}}} A_{t}^{j}\left[\sum_{\substack{Q \in \Gamma \\
L(Q)=k-j+\sigma \\
e^{\varepsilon|k-\tau(Q)|} \leq \mu}} F_{Q}^{j-k}\right]\right| \\
& I V_{s}=\sup _{k} \sup _{\sigma \leq \mu \leq e^{\varepsilon_{1} s}} \sup _{J \in \mathcal{J}_{\mu}^{k}} \sup _{t \in J}\left|\sum_{\substack{\sigma \leq j \leq \mu \\
b_{J}-a_{J} \geq 2^{k-j}}} A_{t}^{j}\left[\sum_{\substack{Q \in \Gamma \\
L(Q)=k-j+\sigma \\
\tau(Q)=k-s}} F_{Q}^{j-k}\right]\right|, \quad s>0, \\
& V_{s}=\sup _{k} \sup _{\sigma \leq \mu \leq e^{\varepsilon_{1}|s|}} \sup _{J \in \mathcal{J}_{\mu}^{k}} \sup _{t \in J}\left|\sum_{\substack{\sigma \leq j \leq \mu \\
b_{J}-a_{J} \geq 2^{k-j}}} A_{t}^{j}\left[\sum_{\substack{Q \in \Gamma \\
L(Q)=k-j+\sigma \\
\tau(Q)=k-s}} F_{Q}^{j-k}\right]\right|, \quad s<0 .
\end{aligned}
$$

Here we may choose $0<\varepsilon_{1}<(d-2) / 2$. We then prove

$$
\begin{aligned}
& \|I I I\|_{L^{p_{d}}}^{p_{d}} \lesssim 2^{-\sigma(d-1)\left(1-p_{d} / 2\right)} \log (2+\sigma)\|F\|_{X^{p_{d}}}^{p_{d}}, \\
& \left\|I V_{s}\right\|_{L^{2}}^{2} \lesssim 2^{-\sigma\left(d-2-\varepsilon_{0}\left(2-p_{d}\right)\right)} 2^{-s\left(d-2-2 \varepsilon_{1}\right)} \alpha^{2-p_{d}}\|F\|_{X^{p_{d}}}^{p_{d}}, \quad s \geq 0, \\
& \left\|V_{s}\right\|_{L^{p_{d}}\left(\mathbb{R}^{d} \backslash \mathcal{W}\right)}^{p_{p^{2}}} \lesssim 2^{-M\left(2-p_{d}\right)(\sigma+\gamma|s|)}\|F\|_{X^{p_{d}}}^{p_{d}}, \quad s<0 .
\end{aligned}
$$

(7.4) follows from (7.5), (7.6) and (7.7) in the usual way. We remark that our assumption (1.14) is needed for (7.5). For the error terms (7.6), (7.7) we can get away with just the regularity hypothesis (1.11) and $\left(\mathcal{C}_{p_{d}, \infty}\right)$.

In the proof we shall use arguments that occur in the proof of Hardy's inequality (see [5]).

Proof of (7.5). We further split $I I I=\sum_{2^{n} \geq \sigma} I I I_{n}$ where

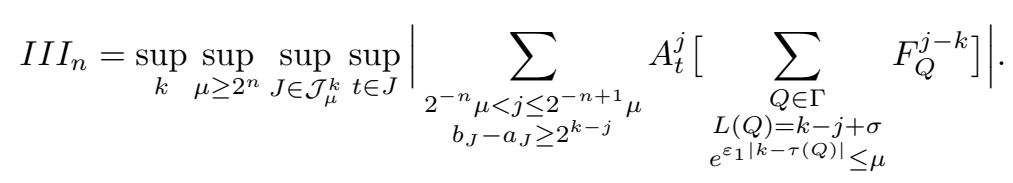


We replace various sup's by $\ell^{p_{d}}$ norms and use Lemma (2.3), (ii). We obtain

$$
\begin{gathered}
\left\|I I I_{n}\right\|_{L^{p_{d}}}^{p_{p}} \leq \sum_{k} \sum_{\mu \geq 2^{n}} \sum_{J \in \mathcal{J}_{\mu}^{k}}\left[\sum_{2^{-n}} \sum_{\mu<j \leq 2^{-n+1} \mu} 2^{(j-\mu) / p_{d}} 2^{-j(d-1) / p_{d}^{\prime}} \operatorname{card}(J)^{1 / p_{d}} 2^{-\sigma(d-1)\left(1 / p_{d}-1 / 2\right)}\right. \\
\left.\times\left(\sum_{\substack{Q \in \Gamma \\
L(Q)=k-j+\sigma \\
|k-\tau(Q)| \leq \varepsilon_{1}^{-1} \log \mu}}|Q|^{1-p_{d} / 2}\left\|F_{Q}^{j-k}\right\|_{L^{2}}^{p_{d}}\right)^{1 / p_{d}}\right]^{p_{d}} .
\end{gathered}
$$

If we abbreviate

$$
\begin{aligned}
& w_{\mu, k}=2^{-\mu} \sum_{J \in \mathcal{J}_{\mu}^{k}} \operatorname{card} J, \\
& b_{Q, \sigma}=|Q|^{1-p_{d} / 2}\left\|F_{Q}^{\sigma-L(Q)}\right\|_{L^{2}}^{p_{d}},
\end{aligned}
$$

this yields

$$
\begin{aligned}
& \left\|I I I_{n}\right\|_{L^{p_{d}}}^{p_{d}} \\
& \lesssim 2^{-\sigma(d-1)\left(1-p_{d} / 2\right)} \sum_{k} \sum_{\mu \geq 2^{n}} w_{k, \mu}\left[\sum_{2^{-n}} \sum_{\mu<j \leq 2^{-n+1} \mu}\left(\sum_{\substack{Q \in \Gamma \\
L(Q)=k-j+\sigma \\
|k-\tau(Q)| \leq \varepsilon_{1}^{-1} \log \mu}} b_{Q, \sigma}\right)^{1 / p_{d}}\right]^{p_{d}} \\
& \lesssim 2^{-\sigma(d-1)\left(1-p_{d} / 2\right)} \sum_{k} \sum_{\mu \geq 2^{n}} w_{k, \mu} \mu^{p_{d}-1} \sum_{2^{-n}} \sum_{\mu<j \leq 2^{-n+1} \mu} \sum_{\substack{L(Q)=k-j+\sigma \\
|k-\tau(Q)| \leq \varepsilon_{1}^{-1} \log \mu}} b_{Q, \sigma} \sum_{\substack{k:|k-\tau(Q)| \leq \varepsilon_{1}^{-1} \log \left(2^{n}(k-L(Q)+\sigma)\right) \\
\mu: 2^{n-1}(k-L(Q)+\sigma) \leq 2^{n}(k-L(Q)+\sigma)}} w_{k, \mu} \mu^{p_{d}-1} b_{Q, \sigma}
\end{aligned}
$$

Now by the regularity assumption $\left(\widetilde{R}_{p_{d}}\right)$ and by $(1.14)$ we have

$$
\begin{aligned}
& \sum_{\substack{\mu: 2^{n-1}(k-L(Q)+\sigma) \leq \\
\mu \leq 2^{n}(k-L(Q)+\sigma)}} w_{k, \mu} \mu^{p_{d}-1} \\
& \quad \lesssim 2^{n\left(p_{d}-1\right)}(k-L(Q)+\sigma+1)^{p_{d}-1} N\left(E^{k}, 2^{k-2^{n}(k-L(Q)+\sigma)}\right) 2^{-2^{n}(|k-L(Q)|+\sigma)} \\
& \quad \lesssim\left[\log \log \left(2^{2^{n}(|k-L(Q)|+\sigma)}\right)\right]^{-1}
\end{aligned}
$$

and thus the expression (7.9) is controlled by

$$
\begin{aligned}
& 2^{-\sigma(d-1)\left(1-p_{d} / 2\right)} 2^{-n\left(p_{d}-1\right)} \sum_{Q} b_{Q, \sigma} \sum_{k:|k-\tau(Q)| \leq \varepsilon_{1}^{-1}(n+\log (k-L(Q)+\sigma))}(1+n+\log (|k-L(Q)|+\sigma))^{-1} \\
& \lesssim 2^{-\sigma(d-1)\left(1-p_{d} / 2\right)} 2^{-n\left(p_{d}-1\right)}(\log (2+\sigma)+n) \sum_{Q} b_{Q, \sigma} .
\end{aligned}
$$

Hence

$$
\left\|I I I_{n}\right\|_{L^{p_{d}}}^{p_{d}} \lesssim 2^{-\sigma(d-1)\left(1-p_{d} / 2\right)} 2^{-n\left(p_{d}-1\right)}(\log (2+\sigma)+n)\|F\|_{X_{p_{d}}^{p_{d}}}
$$

which yields the asserted bound (7.5). 
Proof of (7.6). We estimate $I V_{s} \leq \sum_{2^{n}>\sigma} I V_{s, n}$ where

$$
I V_{s, n}=\sup _{k} \sup _{\sigma \leq \mu \leq e^{\varepsilon_{1} s}} \sup _{J \in \mathcal{J}_{\mu}^{k}} \sup _{t \in J}\left|\sum_{\substack{2^{-n} \\
b_{J}-a_{J} \geq 2^{k-j}}} \mathcal{A}_{t}^{j}\left[\sum_{\begin{array}{c}
Q \in \Gamma \\
L(Q)=k-j+\sigma \\
k-\tau(Q)=s
\end{array}} F_{Q}^{j-k}\right]\right| .
$$

We apply Hölder's inequality for the sum in $j$ and apply Lemma 2.3 to get

$$
\begin{aligned}
\left\|I V_{s, n}\right\|_{L^{2}}^{2} & \lesssim \sum_{k} \sum_{\sigma \leq \mu \leq e^{\varepsilon_{1} s}} \sum_{J \in \mathcal{J}_{\mu}^{k}} 2^{-n}(\mu+1) \sum_{\begin{array}{c}
2^{-n} \\
b_{J}-a<j \leq 2^{-n+1} \mu 2^{k-j}
\end{array}}\left\|\sup _{t \in J}\left|\mathcal{A}_{t}^{j}\left[\sum_{\substack{Q \in \Gamma \\
L(Q)=k-j+\sigma \\
k-\tau(Q)=s}} F_{Q}^{j-k}\right]\right|\right\|_{L^{2}}^{2} \\
& \lesssim 2^{-n} e^{\varepsilon_{1} s} \sum_{k} \sum_{\sigma \leq \mu \leq e^{\varepsilon_{1} s}} \sum_{J \in \mathcal{J}_{\mu}^{k}} \operatorname{card}(J) \sum_{2^{-n}} \sum_{\mu<j \leq 2^{-n+1} \mu} 2^{-j(d-2)} 2^{-\mu} \sum_{\substack{Q \in \Gamma \\
L(Q)=k-j+\sigma \\
k-\tau(Q)=s}}\left\|F_{Q}^{j-k}\right\|_{L^{2}}^{2} .
\end{aligned}
$$

Now we use $2^{L(Q) d\left(1 / p_{d}-1 / 2\right)}\left\|F_{Q}^{j-k}\right\|_{L^{2}} \lesssim 2^{\varepsilon_{0} \sigma} 2^{\tau(Q)(d-1)} \alpha$ and that $k=\tau(Q)+s, j=\tau(Q)-L(Q)+s+\sigma$ and derive

$$
\begin{aligned}
\left\|I V_{s, n}\right\|_{L^{2}}^{2} \lesssim 2^{-n} e^{\varepsilon_{1} s} & \sum_{\substack{Q \in \Gamma \\
\tau(Q)-L(Q) \geq-s}}\left\|F_{Q}^{\sigma-L(Q)}\right\|_{L^{2}}^{p_{d}} 2^{\varepsilon_{0} \sigma\left(2-p_{d}\right)} 2^{\tau(Q)(d-1)\left(2-p_{d}\right)} \alpha^{2-p_{d}} \\
& \times 2^{-(\tau(Q)-L(Q)+s+\sigma)(d-2)}\left\{\sum_{\substack{\sigma \leq \mu \leq e^{\varepsilon_{1} s} \\
\mu \leq 2^{n}(\tau(\bar{Q})-L(Q)+s+\sigma) \\
\mu \geq 2^{n-1}(\tau(Q)-L(Q)+s+\sigma)}} 2^{-\mu} \sum_{\substack{\tau \in \mathcal{J}_{\mu}^{\tau(Q)+s}\\
}} \operatorname{card} J\right\}
\end{aligned}
$$

The expression $\{\ldots\}$ is $O(1)$ by (1.11). We compute that $(d-2)-d\left(1 / p_{d}-1 / 2\right)\left(2-p_{d}\right)=d\left(1-p_{d} / 2\right)$ and $(d-1)\left(2-p_{d}\right)=d-2$. Thus the last estimate simplifies to

$$
\begin{aligned}
\left\|I V_{s, n}\right\|_{L^{2}}^{2} & \lesssim 2^{-n} e^{\varepsilon_{1} s} \sum_{\substack{Q \in \Gamma \\
\tau(Q)-L(Q) \geq-s}} 2^{L(Q) d\left(1-p_{d} / 2\right)}\left\|F_{Q}^{\sigma-L(Q)}\right\|_{L^{2}}^{p_{d}} 2^{\varepsilon_{0} \sigma\left(2-p_{d}\right)} \alpha^{2-p_{d}} 2^{-(s+\sigma)(d-2)} \\
& \lesssim \alpha^{2-p_{d}} 2^{-n} 2^{-\sigma\left(d-2-\varepsilon_{0}\left(2-p_{d}\right)\right)} 2^{-s\left(d-2-2 \varepsilon_{1}\right)} \sum_{Q}|Q|^{1-p_{d} / 2}\left\|F_{Q}^{\sigma-L(Q)}\right\|_{L^{2}}^{p_{d}}
\end{aligned}
$$

which implies (7.6).

Proof of (7.7). This $L^{p_{d}}$ estimate away from the exceptional set follows by analogous arguments; Lemma 2.3(iii) is used. We omit the details.

This completes the proof of Proposition 7.1.

\section{Examples and counterexamples}

We consider a simple class of sets $E$ to which Theorems I-IV can be applied. They satisfy the

8.1. Convexity assumption. For each $k \in \mathbb{Z}$ the set $E^{k}$ is given by $\left\{t_{\nu}^{k}\right\}_{\nu=1}^{\infty}$ where $t_{\nu}^{k}$ is a monotone sequence contained in $\left[2^{k}, 2^{k+1}\right]$, so that the sequence $t_{\nu+1}^{k}-t_{\nu}^{k}$ is also monotone.

The following lemma shows that if $\left(\mathcal{C}_{p, \infty}\right)$ holds for some $p<d /(d-1)$ and $E$ satisfies the convexity assumption; then it also satisfies the regularity assumption for all $p>1$. 
Lemma 8.1.1. Suppose $E$ satisfies the convexity assumption. Suppose that for some $\beta>0$ the estimate

$$
\sup _{k} N\left(E^{k}, 2^{k-n}\right) \leq C \frac{2^{n}}{(1+n)^{\beta}}
$$

holds uniformly in $k \in \mathbb{Z}$. Then $E$ satisfies regularity assumption $\left(\mathcal{R}_{p}\right)$ for all $p>1+[(d-1)(\beta+1)]^{-1}$. Moreover it satisfies regularity assumption $\left(\widetilde{\mathcal{R}}_{d /(d-1)}\right)$.

Proof. We write $E^{k}$ as a sequence $t_{\nu}^{k}$ and let $J_{\mu}^{k}$ consist of those $t \in E^{k}$ where $2^{k-\mu} \leq t_{\nu}^{k}-t_{\nu+1}^{k}<$ $2^{k-\mu+1}$ (assuming without loss of generality that the $t_{\nu}^{k}$ are decreasing in $\nu$ ). We clearly have card $J_{\mu}^{k} \lesssim$ $N\left(E^{k}, 2^{k-\mu}\right)$.

Let $a_{\mu}^{k}$ and $b_{\mu}^{k}$ denote the endpoints of the equally spaced set $J_{\mu}^{k}$. Let $\mathcal{D}^{k}=\cup_{\mu}\left\{a_{\mu}^{k}, b_{\mu}^{k}\right\}$, the set of endpoints. The assertion is implied by the estimate

$$
N\left(\mathcal{D}^{k}, 2^{k-j}\right) \lesssim 2^{j /(1+\beta)} .
$$

Let $L=L_{j}$ be the smallest integer $\geq 2^{j /(1+\beta)}$. Note that the set $\cup_{\mu \geq L_{j}} J_{\mu}^{k}$ is contained in an interval of length

$$
\lesssim N\left(E^{k}, 2^{k-L_{j}}\right) 2^{k-L_{j}} \lesssim 2^{L_{j}}\left(1+L_{j}\right)^{-\beta} 2^{k-L_{j}} \lesssim\left(1+L_{j}\right)^{-\beta} 2^{k} .
$$

This interval can be covered by intervals of length $2^{k-j}$ and we need at most $\left(1+L_{j}\right)^{-\beta} 2^{j}$ such intervals to do this. But $\left(1+L_{j}\right)^{-\beta} 2^{j} \lesssim 2^{j /(1+\beta)}$.

We still need to cover the points in $\mathcal{D}^{k}$ which do not belong to $\cup_{\mu \geq L_{j}} J_{\mu}^{k}$. But $\mathcal{D}^{k}$ consists just of the $a_{\mu}^{k}$ and the $b_{\mu}^{k}$ and there are at most $2 L_{j} \lesssim 2^{j /(1+\beta)}$ points in $\mathcal{D}^{k}$ which are not yet covered. This implies (8.2).

In order to verify the condition (1.11) it suffices to show

$$
\sum_{\mu>n} 2^{-\mu} \operatorname{card}\left(J_{\mu}^{k}\right) \lesssim 2^{-n} N\left(E^{k}, 2^{k-n}\right)
$$

But if $a^{k}=\inf _{\mu} a_{\mu}^{k}=\inf E^{k}$ then the left side of (8.3) is $\approx 2^{-k}\left(b_{n}^{k}-a^{k}\right)$. Moreover every subinterval of length $2^{k-n}$ of $\left[a^{k}, b_{n}^{k}\right]$ contains points in $E^{k}$ and therefore $b_{n}^{k}-a^{k} \lesssim 2^{k-n} N\left(E^{k}, 2^{k-n}\right)$; thus (8.3) holds.

Proof of Theorem 1.1. The set $E^{k}=\left\{2^{k}\left(1+\nu^{-\alpha}\right): \nu \in \mathbb{Z}^{+}\right\}$satisfies $N\left(E^{k}, 2^{k} \delta\right) \lesssim \delta^{1 /(\alpha+1)}$ and assertion (i) follows from Lemma 8.1.1 and Theorem II. On the other hand, the set $E^{k}=\left\{2^{k}\left(1+\log ^{-\beta}(2+\right.\right.$ $\left.\nu): \nu \in \mathbb{Z}^{+}\right\}$satisfies $N\left(E^{k}, 2^{k} \delta\right) \lesssim \delta^{-1}[\log (1 / \delta)]^{-\beta}$ and assertion (ii) follows from Lemma 8.1.1 and Theorem IV.

\subsection{A counter-example to $L^{p}$ boundedness for a related maximal function.}

Let $E_{0}$ be any set in $[1,2]$ and define the modified maximal function

$$
\widetilde{M}_{E_{0}} f(x):=\sup _{r \in E_{0}} f * d \sigma\left(x+r e_{1}\right)
$$

in $\mathbb{R}^{d}$, where $e_{1}$ is a unit vector. If $E_{0}$ satisfies the regularity assumption $\left(\mathcal{R}_{p}\right), p<d /(d-1)$ then the condition $\mathcal{C}_{p, p}$ is necessary and sufficient for $L^{p}$ boundedness of $\widetilde{M}_{E_{0}}$; indeed a notational modification of the proof of Theorem I applies to show the sufficiency. Note that for sets $E_{0}$ supported in [1,2] the conditions $\mathcal{C}_{p, p}$ and $\mathcal{C}_{p, \infty}$ both amount to the inequality $N\left(E_{0}, \delta\right) \lesssim \delta^{-(d-1)(p-1)}$. However $L^{p}$ boundedness and indeed the weak type $(p, p)$ property may fail if we drop the regularity assumption. 
Let $E_{0}$ be the middle-halves Cantor set consisting of all $t=1+\sum_{j=1}^{\infty} b_{j} 4^{-j}$ where $b_{j} \in\{0,2\}$. Then the Minkowski dimension of $E_{0}$ is $1 / 2$ and $\widetilde{M}_{E_{0}}$ is bounded on $L^{p}\left(\mathbb{R}^{2}\right)$ for $p>3 / 2$ and unbounded on $L^{p}\left(\mathbb{R}^{2}\right)$ for $p<3 / 2$. Moreover $\mathcal{C}_{p, p}$ holds for $p=3 / 2$. We show that nevertheless $\widetilde{M}_{E_{0}}$ is not of weak type $(3 / 2,3 / 2)$.

Let $N$ be large and define

$$
f(x):=\sum_{i=1}^{N} 4^{i} \chi_{2 C e_{1}+B\left(0, a 4^{-i}\right)}(x),
$$

where $C$ is the Cantor set $C=\left\{\sum_{j} c_{j} 4^{-j}: j=0,1\right\}$ and $a$ is small. Note that $\|f\|_{3 / 2} \lesssim N^{2 / 3}$ (each $i$ contributes an $L^{3 / 2}$ norm of $O(1)$, and the contributions are mostly disjointly supported).

Now $E_{0}+C$ fills out the interval [1,2] and thus the maximal function $\widetilde{M}_{E_{0}} f$ has size about $N$ on a fixed portion of the unit annulus, thus $\left\|\widetilde{M}_{E_{0}} f\right\|_{L^{3 / 2, \infty}} \geq c N$. This shows that $\widetilde{M}_{E_{0}}$ is not of weak type $(3 / 2,3 / 2)$. A closer examination shows that $f$ belongs to the Lorentz space $L^{3 / 2, s}$ with norm $O\left(N^{s}\right)$ so that $\widetilde{M}_{E_{0}}$ fails to map the Lorentz space $L^{3 / 2, s}$ to $L^{3 / 2, \infty}$ when $s>1$. Unfortunately this example is too rigid in order to apply to the maximal operator $\mathcal{M}_{E_{0}}$ considered in this paper.

8.3. Failure of restricted weak type $(2,2)$ in two dimensions. We shall now turn to the counterexample mentioned in the introduction and give a proof of Proposition 1.5.

Suppose that there is a large constant $B$ so that there exists $k$ and $n \geq 100$ such that

$$
N\left(E^{k}, 2^{k-2 n}\right) \geq B 2^{2 n} / n
$$

We then show that $\left\|\mathcal{M}_{E}\right\|_{L^{2,1} \rightarrow L^{2, \infty}} \geq c \sqrt{B}$ for some absolute constant $c$. By rescaling we may assume $k=0$ and $n \gg 1$.

We use the construction of a Kakeya set as given by Keich [6], rescaled to a square of sidelength $2^{-n}$. It gives us $\approx 2^{n}$ rectangles $R_{l}$ with sidelengths $2^{-n-3}$ and $2^{-2 n-6}$ so that $R_{l} \subset\left[-2^{-n}, 2^{-n}\right]^{2}$ and the longer side of $R_{l}$ is parallel to $e_{l}:=\left(\cos l 2^{-n}, \sin l 2^{-n}\right)$, and the union $A=\cup R_{l}$ has measure $\lesssim 2^{-2 n} n^{-1}$. Thus $\left\|\chi_{A}\right\|_{L^{2,1}} \approx\left\|\chi_{A}\right\|_{2} \lesssim 2^{-n} n^{-1 / 2}$.

Let $\left\{I_{\nu}\right\}_{\nu=1}^{N}$ be a cover of the set $E^{0}$ by dyadic intervals of length $2^{-2 n}$, with disjoint interior so that $N \geq B 2^{2 n} / n$. Let $I_{\nu}=\left[a_{\nu}, b_{\nu}\right]$, and assume $a_{\nu}<a_{\nu+1}$. We then pick every tenth interval $=I_{10 \nu}$, moreover we pick every tenth rectangle $R_{10 l}$ in the above Kakeya construction. Let $e_{l}^{\perp}:=\left(-\sin l 2^{-n}, \cos l 2^{-n}\right)$ and let $R_{l, \nu}$ be the translate $a_{10 \nu} e_{10 l}^{\perp}+R_{10 l}$. Then the rectangles $R_{l, \nu}$ are disjoint, however on a tenth fraction of each of these rectangles we have that $\mathcal{M}_{E} \chi_{A}(x)>c 2^{-n}$. There are $\approx N 2^{n} / 100$ such rectangles and thus

$$
\operatorname{meas}\left(\left\{x: \mathcal{M}_{E} \chi_{A}(x)>c 2^{-n}\right\}\right) \geq c^{\prime} N 2^{n} 2^{-3 n} \gtrsim B n^{-1}
$$

but on the other hand $\left\|\chi_{A}\right\|_{2}^{2} /\left(2^{-2 n}\right) \lesssim n^{-1}$ so that the $L^{2,1} \rightarrow L^{2, \infty}$ operator norm is $\gtrsim \sqrt{B}$. This proves the proposition.

\section{REFERENCES}

1. J. Bourgain, Estimations de certaines fonctions maximales, C. R. Acad. Sc. Paris, 310 (1985).

2. _ـ Averages in the plane over convex curves and maximal operators, Jour. Anal. 47 (1986), 69-85.

3. S.Y.A. Chang and R. Fefferman, The Calderón-Zygmund decomposition on product domains, Amer. J. Math 104 (1982), 445-468.

4. M. Christ, Weak type (1,1) bounds for rough operators, Annals of Math. 128 (1988), 19-42.

5. G. Hardy, J.E. Littlewood and G. Pólya, Inequalities, Cambridge Univ. Press, Cambridge, 1952.

6. U. Keich, On $L^{p}$ bounds for Kakeya maximal functions and the Minkowski dimension in $\mathbb{R}^{2}$, Bull. London Math. Soc 31 (1999), 213-221. 
7. M. Leckband, A note on the spherical maximal operator for radial functions, Proc. Amer. Math. Soc. 100 (1987), 635-640.

8. J. Peetre, On spaces of Triebel-Lizorkin type, Ark. Mat. 13 (1975), 123-130.

9. A. Seeger, Remarks on singular convolution operators, Studia Math. 97 (1990), 91-114.

10. A. Seeger and T. Tao, Sharp Lorentz space estimates for rough operators, Math. Annalen 320 (2001), 381-415.

11. A. Seeger, T. Tao and J. Wright, Singular maximal functions and Radon transforms near $L^{1}$, preprint.

12. A. Seeger, S. Wainger and J. Wright, Pointwise convergence of spherical means, Math. Proc.Cambr. Phil. Soc. 118 (1995).

13. - Spherical maximal operators on radial functions, Math. Nachr. 187 (1997), 95-105.

14. E. M. Stein, Maximal functions: spherical means, Proc. Nat. Acad. Sci. 73 (1976), 2174-2175.

15. __ Harmonic analysis: Real variable methods, orthogonality and oscillatory integrals, Princeton Univ. Press, Princeton, 1993.

16. T. Wolff, Recent work connected with the Kakeya problem, Prospects in Mathematics (Princeton, N.J., 1996), Amer. Math. Soc., Providence, R.I., 1999, pp. 129-162. 